\title{
The combination of loss of glyoxalase1 and obesity results in hyperglycemia
}

\author{
Elisabeth Lodd, ${ }^{1}$ Lucas M. Wiggenhauser, ${ }^{1}$ Jakob Morgenstern, ${ }^{2}$ Thomas H. Fleming, ${ }^{2}$ \\ Gernot Poschet, ${ }^{3}$ Michael Büttner, ${ }^{3}$ Christoph T. Tabler, ${ }^{1}$ David P. Wohlfart, ${ }^{1}$ Peter P. Nawroth, ${ }^{2,4,5}$ \\ and Jens Kroll ${ }^{1}$ \\ 'Department of Vascular Biology and Tumor Angiogenesis, European Center for Angioscience (ECAS), Medical Faculty \\ Mannheim, Heidelberg University, Mannheim, Germany. ${ }^{2}$ Department of Internal Medicine I and Clinical Chemistry, \\ Heidelberg University Hospital, Heidelberg, Germany, ${ }^{3}$ Metabolomics Core Technology Platform, Centre for Organismal \\ Studies, Heidelberg University, Heidelberg, Germany, ${ }^{4}$ German Center for Diabetes Research (DZD), München-Neuherberg, \\ Germany, 5Joint Heidelberg-IDC Translational Diabetes Program, Helmholtz-Zentrum, München, Heidelberg, Germany.
}

The increased formation of methylglyoxal (MG) under hyperglycemia is associated with the development of microvascular complications in patients with diabetes mellitus; however, the effects of elevated MC levels in vivo are poorly understood. In zebrafish, a transient knockdown of glyoxalase 1, the main MG detoxifying system, led to the elevation of endogenous MG levels and blood vessel alterations. To evaluate effects of a permanent knockout of glyoxalase 1 in vivo, glo1//- zebrafish mutants were generated using CRISPR/Cas9. In addition, a diet-induced-obesity zebrafish model was used to analyze $\mathrm{glo1}^{-/-}$zebrafish under high nutrient intake. Glo1//- zebrafish survived until adulthood without growth deficit and showed increased tissue MC concentrations. Impaired glucose tolerance developed in adult $\mathrm{glo1^{-/- }}$ zebrafish and was indicated by increased postprandial blood glucose levels and postprandial $\mathbf{S 6}$ kinase activation. Challenged by an overfeeding period, fasting blood glucose levels in glo1/- zebrafish were increased which translated into retinal blood vessel alterations. Thus, the data have identified a defective MG detoxification as a metabolic prerequisite and glyoxalase 1 alterations as a genetic susceptibility to the development of type 2 diabetes mellitus under high nutrition intake.

Conflict of interest: The authors have declared that no conflict of interest exists.

Copyright: (c) 2019 American Society for Clinical Investigation

Submitted: November 12, 2018 Accepted: May 16, 2019 Published: June 20, 2019

Reference information: /CI Insight. 2019;4(12):e126154. https://doi. org/10.1172/jci.insight.126154.

\section{Introduction}

Diabetes mellitus affects more than 451 million people worldwide and is attributable for 5 million deaths in 2017 (1). The disease is characterized by hyperglycemia, with $90 \%$ of diabetic patients being affected by type 2 diabetes mellitus. The prevalence of type 2 diabetes mellitus in the past 2 decades has raised dramatically because of an increase in risk factors, such as obesity and overweight (2). Type 2 diabetes mellitus is initiated by peripheral insulin resistance and hyperinsulinemia, finally leading to diminution in insulin secretion (3). Diabetic patients experience microvascular and macrovascular complications that can cause blindness, kidney failure, and amputations. Although lowering blood glucose levels can decrease the incidence of diabetic complications in some patients, meta-analysis of randomized controlled trials imply that intensive glycemic control cannot reduce the risk of microvascular complications in patients with type 2 diabetes mellitus (4).

Methylglyoxal (MG) is a reactive metabolite that modifies amino acid residues of proteins, lipids, and DNA and is a main precursor of advanced glycation end products (AGEs) (5). Under physiological conditions, MG is mainly detoxified by the glyoxalase system that is present in the cytosol of all mammalian cells (6). The degradation of MG consists of 2 steps, the first catabolized by glyoxalase 1, converts MG with the cofactor glutathione to the nontoxic metabolite S-D-lactoylglutathione. S-D-lactoylglutathione is further degraded to D-lactate by glyoxalase 2 (7). In addition to the glyoxalase system, aldo-keto reductase and aldehyde dehydrogenase can detoxify MG $(8,9)$. Recently, aldo-keto reductase and aldehyde dehydrogenase could be shown to efficiently compensate for loss of glyoxalase 1 in cells and mice $(10,11)$.

MG is elevated in the plasma and tissue of diabetic patients $(6,12-14)$ and AGEs are strongly linked to the development of microvascular complications including retinopathy $(15,16)$, nephropathy $(17,18)$, and neuropathy (19). In addition, MG is associated with the initiation and progression of type 2 diabetes mellitus through the impairment of insulin signaling $(20,21)$, insulin secretion $(22,23)$, and pancreatic beta cell function (24). 
Although these findings indicate the important role of $\mathrm{MG}$ in the pathogenesis of diabetes mellitus, most studies have only focused on external addition of MG (24) to cells or rodent models; however, the consequences of endogenous MG elevation are still poorly understood. Recently, glo1-knockout flies and mice were reported (11, 25, 26). Glo1-knockout mice compensate effectively for the loss of glyoxalase 1, whereas the glo1-knockout fly has elevated MG concentrations. This endogenous elevation of MG in flies leads to increased fatty acid synthase activity and with increasing age to obesity, insulin resistance, and increased hemolymph glucose levels (25).

The zebrafish has evolved as a model for diabetes research in the past decade $(27,28)$. Glucose homeostasis in zebrafish is very similar to humans and other mammals and glucose-induced alterations of the retina (29), kidney (30), and neuronal tissue (31) have been shown to develop in adult zebrafish. We have previously shown that high tissue levels of MG, induced by the external addition of MG or by a transient knockdown of the glyoxalase 1 in zebrafish larvae, altered intersegmental blood vessels in the zebrafish embryo through the activation of angiogenic signal cascades (32). However, it remained unclear if a permanent knockout of glyoxalase 1 leads to increased MG concentrations in juvenile and adult zebrafish and if $\mathrm{glol}^{-1-}$ zebrafish show organ alterations.

Therefore, the aim of the study was to evaluate long-term effects of a glyoxalase 1 knockout on glu-

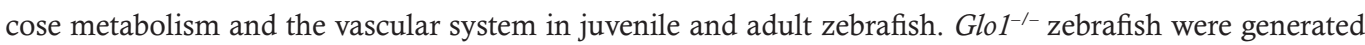
using the CRISPR/Cas9 technology and $\mathrm{glo1}^{-/-}$zebrafish displayed increased tissue MG concentrations and impaired glucose tolerance as indicated by transiently increased postprandial blood glucose levels and postprandial S6 kinase activation. Overfed adult $\mathrm{glo1}^{-1-}$ zebrafish showed fasting hyperglycemia accompanied by retinal vascular alterations. Thus, our data suggest an impaired MG detoxification as a marker for the genetic susceptibility to the development of type 2 diabetes mellitus.

\section{Results}

Generation of glo1 ${ }^{-1-}$ zebrafish using CRISPR-Cas9-mediated genome editing. To investigate long-term effects of a compromised MG metabolism in vivo, we generated a knockout zebrafish line for Glo1, an enzyme that is part of the main MG detoxifying system in cells (7). Therefore, we designed a CRISPR-gRNA targeting exon 3 of glo1 (Figure 1A) using the ZiFit-targeter Version 3.3 and injected the glo1 gRNA with Cas9mRNA in zebrafish embryos (33). After crossing the F0-positive mosaic mutants with wild-type line, we identified different mutations in $g l o 1$ and selected 2 frameshift mutations, namely deletions of 5 (delta5) and 8 (delta 8) nucleotides (Figure 1A), for further breedings and experiments. Both mutations in glo1 led to a total loss of Glo1 enzyme activity (Figure 1B) and Glol protein expression (Figure 1C) in glo $^{-{ }^{--}}$zebrafish larvae. Glo1 $^{-/-}$zebrafish larvae survived, showed no growth retardation effects (Figure 1D), and had 1.5-fold higher tissue MG concentrations than $g l o 1^{+/+}$zebrafish larvae at 96 hours postfertilization (hpf) (Figure 1E), whereas 3-deoxyglucosone (3-DG) and glyoxal levels were unaltered (Supplemental Figure 1A; supplemental material available online with this article; https://doi.org/10.1172/jci.insight.126154DS1). Although deficits in MG metabolism are associated with altered glutathione metabolism, the ratio of reduced to oxidized glutathione was unaltered in $g l o 1^{-/-}$zebrafish (Supplemental Figure 1B). In addition, Glo1 knockout did not alter energy and reduction equivalent levels in zebrafish larvae (Supplemental Figure 2). These results showed for the first time to our knowledge the generation of $\mathrm{glo}^{1^{--}}$zebrafish and that $\mathrm{glol}^{-{ }^{--}}$zebrafish are viable and fertile, despite increased tissue MG levels.

Vascular development of glo1 $^{-1-}$ zebrafish larvae is unaltered as a result of activation of different compensatory mechanisms. We have previously shown that elevated tissue MG levels, achieved through external addition of MG or the transient knockdown of Glo1 using a morpholino-based approach, induced altered formation of the trunk vasculature in zebrafish larvae (32). First, we evaluated whether a permanent knockout of Glo1 has the same effect on vascular development as the transient knockdown of Glo1. To address this question, we used the $T G(f l i 1: E G F P)$ vascular reporter line carrying a mutation in the glo1 gene to analyze the zebrafish vasculature. The results showed no induction of ectopic blood vessel formation in $\mathrm{glo1}^{-1-}$ zebrafish larvae compared with $\mathrm{glo1}^{+/+}$zebrafish larvae at $96 \mathrm{hpf}$ (Figure 2, A and B). Next, we asked the question of whether the vasculature of $\mathrm{glol}^{-1-}$ zebrafish larvae is more susceptible to high MG concentrations. After incubation of $\mathrm{glo}^{+/+}$zebrafish larvae with $500 \mu \mathrm{M} \mathrm{MG}$, the number of hyperbranches was 3- to 4-fold increased, whereas a Glo1 knockout could not further aggravate the MG-driven hyperbranch formation (Figure 2, A and B). These data, together with the only 1.5-fold increase of MG in the $\mathrm{glo1}^{1^{--}}$zebrafish larvae (Figure $1 \mathrm{E}$ ), indicate compensatory mechanisms for the Glo1 knockout in zebrafish by the upregulation of alternative MG-detoxifying enzymes $(8,9)$. 

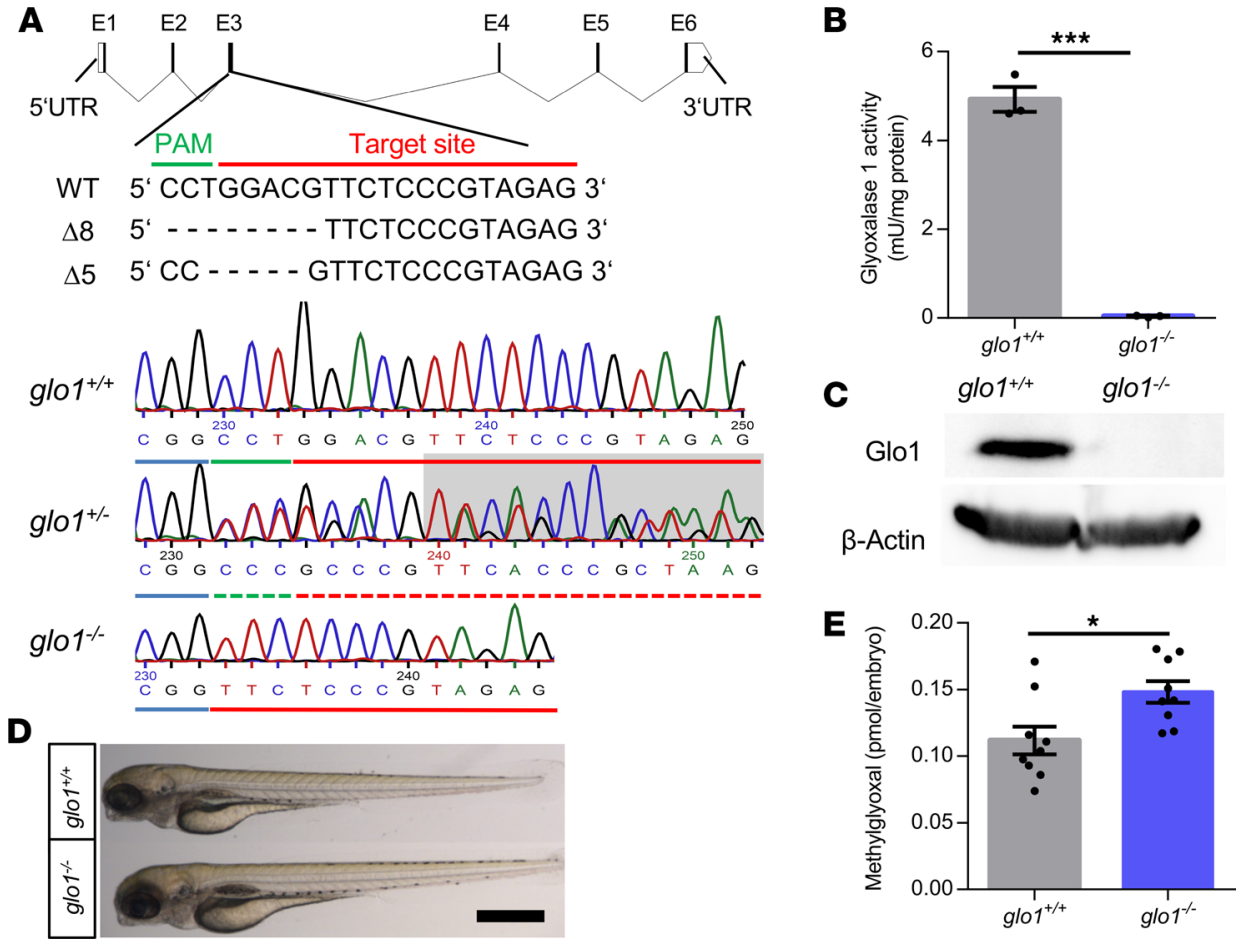

Figure 1. Generation of glo1 $^{-/-}$zebrafish using CRISPR-Cas9 technology. (A) Glo1-CRISPR-target site was designed in exon 3 of glo1 and CRISPR/Cas9-induced deletions of 5 and 8 nucleotides were selected for further Clo1 mutant line generation and maintenance. Genotype was analyzed using sequencing chromatograms of PCR-amplified glo1 region, containing the glo1 target site. Chromatogram shows glo1 wild type, heterozygous, and homozygous sequencing results. (B) $\mathrm{g} / 01^{-/-}$zebrafish show no Clo1 enzyme activity measured by spectrophotometric analysis in zebrafish lysates at 96 hpf; $n=3$ clutches with 35 to 50 larvae; mean \pm SEM. (C) Representative Western blot shows

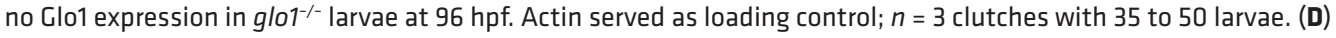
Microscopic images show normal gross morphology of $\mathrm{glo1^{-/- }}$ larvae at $96 \mathrm{hpf}$ in comparison with $\mathrm{glo1^{+/+ }}$ larvae. Black

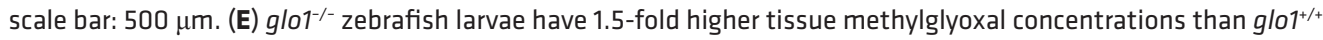
zebrafish. Methylglyoxal levels were determined by LC-MS/MS in zebrafish lysates at 96 hpf; $n=9$ clutches with 35 larvae; mean \pm SEM. For statistical analysis Student's $t$ test $(\mathbf{B})$ and Mann-Whitney $U$ test $(\mathbf{E})$ were applied. ${ }^{*} P<$ $0.05,{ }^{* *} P<0.001$. PAM, protospacer-adjacent motif; UTR, untranslated region; E, exon; WT, wild type.

In mammalian Schwann cells, members of Akr family and Aldh family compensate for the loss of Glo1 (10). Thus, we hypothesized a compensatory upregulation of these enzyme families and consequently determined the activity of Akr and Aldh in glo $^{-/-}$and Glo1-morpholino-injected zebrafish embryos (Glo1-morphants) at $72 \mathrm{hpf}$. A significant increase of Aldh enzyme activity in glo $^{-/-}$zebrafish larvae was observed, whereas Akr enzyme activity remained unaltered in glo $^{-1^{-/}}$animals compared with $g l o 1^{+/+}$zebrafish embryos (Figure 3, A and B). Glo1-morphants, however, showed no induction of Aldh or Akr enzyme activity in comparison with control morpholino-injected zebrafish embryos (Figure 3, A and B). This finding shows that upregulation of compensatory pathways occurs only in long-term $g l o 1^{-/-}$knockout animal experiments but not in short-term and transient glo $^{-1-}$ knockdown experiments. To identify the aldh family members, which are responsible for increased Aldh enzyme activity, RT-qPCR analyses with tissue lysates of zebrafish embryos at $72 \mathrm{hpf}$ were performed. Results revealed an induction of aldh3a1, aldh3a2a, aldh9a1a2, and aldh9a1b mRNA expression in $\mathrm{glol}^{-/-}$compared with $\mathrm{glo}^{+/+}$zebrafish embryos (Supplemental Figure 3). Together, the data showed an upregulation of Aldh activity and expression in $g l o 1^{-1-}$ zebrafish, which could explain the only 1.5 -fold increase of $\mathrm{MG}$ in $g l o 1^{-1-}$ mutants. This may also explain the missing vascular alterations in $\mathrm{glo1}^{-1-}$ zebrafish as observed in Glo1-morphants (32).

Increased MG levels are associated with mitochondrial dysfunction and increased ROS formation (34, 35). Stearic acid, a long-chain fatty acid, has been shown to be beneficial for mitochondria fusion in flies, and this finding could be recently translated to humans $(36,37)$. In addition, eicosapentaenoic acid, an omega-3 fatty acid, was shown to have a beneficial effect in diabetic kidney disease in mice by suppressing ROS generation and mitochondrial apoptosis (38). Fish, unlike mammals, have high contents of omega-3 fatty acids (39) 
A

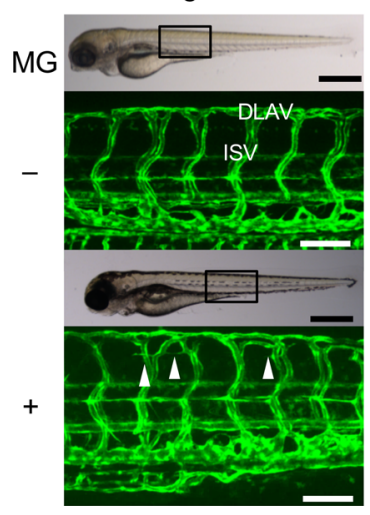

glo $1^{-1-}$

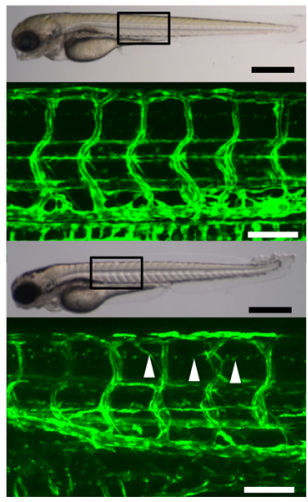

B

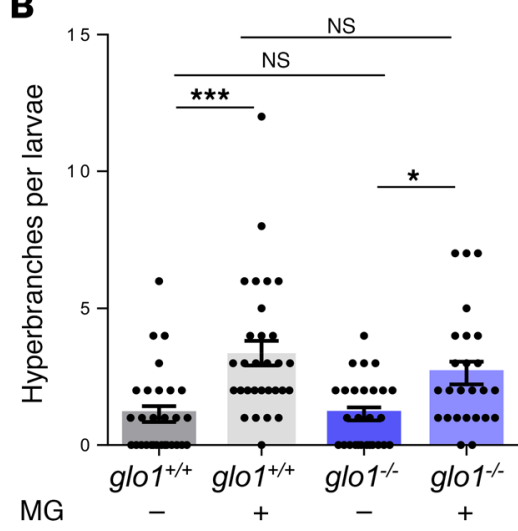

Figure 2. Loss of Glo1 could not aggravate the MG-induced blood vessel hyperbranch formation in zebrafish larvae. (A) Loss of Glo1 enzyme activity has no effect on trunk vessel morphology, whereas incubation in $500 \mu \mathrm{M}$ MG leads to increased formation of hyperbranches (white arrowheads) in the zebrafish trunk vasculature of $\mathrm{glo0^{-/- }}$ and $\mathrm{glo \textrm {T } ^ { + / + }}$ larvae at $96 \mathrm{hpf}$. Light microscopic images show gross morphology of zebrafish larvae and black boxes indicate region seen in the confocal images. White scale bar: $100 \mu \mathrm{m}$. Black scale bar: $500 \mu \mathrm{m}$. (B) Quantification of hyperbranch formation; $n=29-31$ larvae $\left(\mathrm{glot}^{+/+}\right)$and $n=25-27$ larvae $\left(\mathrm{glo1^{-/- }}\right)$, mean \pm SEM. For statistical analysis 1-way ANOVA followed by Sidak's multiple-comparisons test was applied. ${ }^{*} P<0.05,{ }^{* * *} P<0.001$. DLAV, dorsal longitudinal anastomotic vessel; ISV, intersegmental vessel; MG, methylglyoxal; ns, not significant.

and may therefore use this as a protection mechanism against increased MG formation. To test this hypothesis, we performed GC-MS analysis of $\mathrm{glol}^{1^{--}}$zebrafish larvae at $96 \mathrm{hpf}$. The results showed that long-chain unsaturated as well as saturated fatty acid levels were significantly elevated in $\mathrm{glol}^{-/-}$compared with $\mathrm{glo1^{+/+ }}$ zebrafish larvae (Figure 4, A-D). The strongest effect was seen for omega-3 fatty acids, which showed 1.5- to 2-fold higher levels in $\mathrm{glol}^{1^{--}}$than in $\mathrm{glol}^{+/+}$zebrafish larvae (Figure 4A). Although fatty acid metabolism was altered in glo1 $^{-1-}$ zebrafish larvae, citric acid cycle intermediates, sugars, and glycolysis intermediates were unaltered (Supplemental Figure 4). Thus, the data identified increased formation of long-chain saturated and unsaturated fatty acids as a possible metabolic compensatory mechanism present in zebrafish. To clarify the higher abundance of identified fatty acids, we tested the hypothesis that fatty acid synthase and other enzymes important for the synthesis of long-chain fatty acids are higher expressed in $\mathrm{glol}^{1^{--}}$zebrafish. Using a RT-qPCR-based approach, expression analyses of fatty acid synthase, fatty acid desaturase 2 , fatty acid elongase 2 , and stearoyl-CoA desaturase were performed. Yet, we could not show an altered expression of these enzymes induced by a Glo1 knockout (Figure 4E); this finding suggested increased enzymatic activities, potentially induced by posttranslational modifications, as one mechanism leading to higher concentrations of long-chain unsaturated as well as saturated fatty acids (25). Altogether, these data identified Aldh and elevated fatty acid levels as compensatory pathways for loss of Glo1 in zebrafish larvae, which nevertheless were not able to maintain MG levels as seen in wild-type zebrafish larvae.

Hyperglycemia develops in diet-induced obese glo1 $1^{-1}$ zebrafish. The increased formation of MG is associated with the formation of late diabetic complications and the development of insulin resistance (19-21, 40). Thus, we have studied the long-term effects of a Glo1 knockout on zebrafish. We analyzed adult zebrafish regarding their MG concentrations and blood glucose levels. Glo1 ${ }^{-1-}$ adult zebrafish showed no growth deficit and had a normal gross morphology compared with $g l o 1^{+/+}$zebrafish (Figure 5A). In line with $\mathrm{glo1}^{1^{--}}$zebrafish larvae, whole body lysates of juvenile $\mathrm{glo1^{-1- }}$ showed 1.5-fold higher MG levels than $\mathrm{glol}^{+/+}$zebrafish (Figure 5B). The fasting blood glucose level of $\mathrm{glol}^{-/-}$and $\mathrm{glol}^{+/+}$zebrafish was around 30 to $40 \mathrm{mg} / \mathrm{dL}$ (Figure 5C), which is in line with published data for zebrafish (41, 42). One hour after feeding, the postprandial blood glucose level in $\mathrm{glo1}^{-1-}$ zebrafish was highly increased, reaching levels of $118 \mathrm{mg} / \mathrm{dL}$ compared with a blood glucose level of $65 \mathrm{mg} / \mathrm{dL}$ in $g l o 1^{+/+}$zebrafish (Figure 5D). Yet, after 2 additional hours, $g l o 1^{+/+}$as well as $\mathrm{glol}^{-{ }^{--}}$zebrafish achieved blood glucose levels similar to the fasting state (Figure 5, E and C). To evaluate whether the liver metabolism and metabolic stress responses are affected by Glo1 knockout, livers were dissected 1 hour postprandial and L-lactate, glycogen, AGEs, and oxidation products were determined. Although there was a tendency toward a decrease in glycogen and an increase in L-lactate levels in $\mathrm{glo}^{1^{-/}}$compared with $\mathrm{glo}^{+/+}$zebrafish liver lysates, the differences were 
A

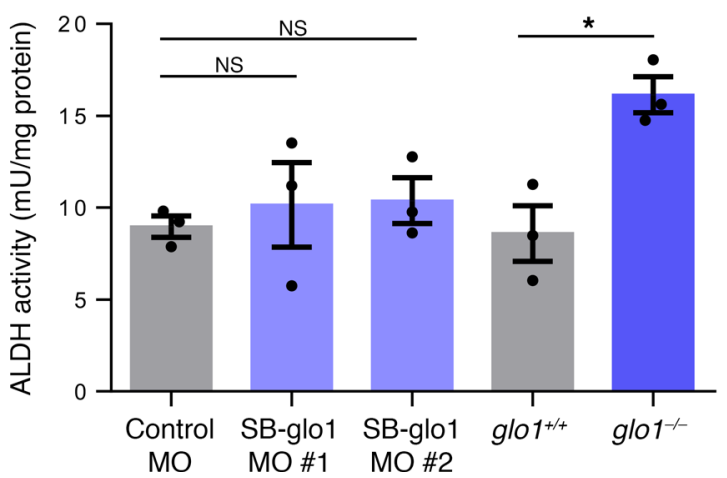

B

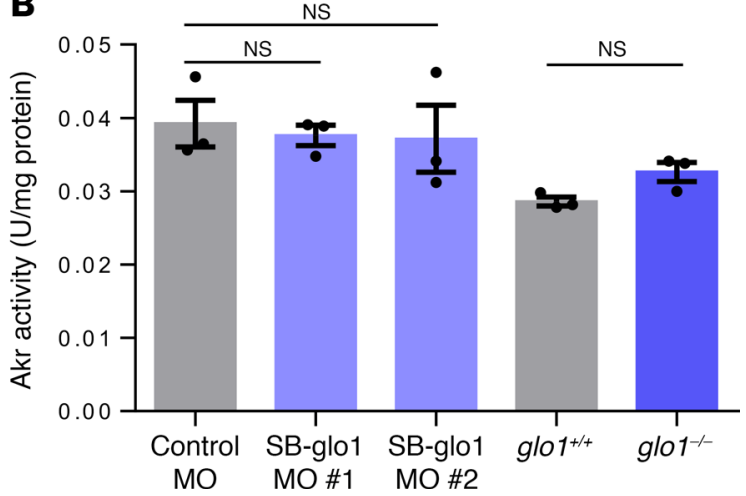

Figure 3. Aldh activity, but not Akr activity, partially compensate for loss of Glo1 enzyme activity in zebrafish larvae. (A) Aldh enzyme activity is induced by Glo1 knockout in glo1/- zebrafish larvae but not by morpholino-mediated Glo1 knockdown. Three nanograms of morpholinos SB-glo1M0\#1, SB-glo1MO\#2, and ControlMO were injected into the 1-cell stage of zebrafish embryos and analyzed at 72 hpf. (B) Akr enzyme activity remains unaltered

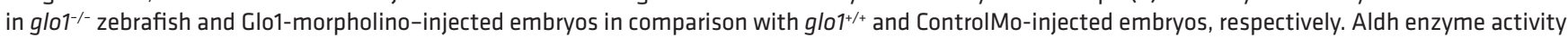
in (A) and Akr enzyme activity in (B) were determined in zebrafish larvae lysates at 72 hpf by spectrophotometric analysis; $n=3$ clutches with 35 to 50 larvae per group; mean \pm SEM. For statistical analysis 1-way ANOVA followed by Tukey's multiple-comparisons test was applied. ${ }^{*} P<0.05$. Aldh, aldehyde dehydrogenase; Akr, aldo-keto reductase; MO, morpholino; ns, not significant.

not significant (Supplemental Figure 5, A and B). In addition, AGEs and protein oxidation marker dityrosine and methionine sulphoxide were unaltered in $\mathrm{glo1}^{-1-}$ zebrafish liver lysates (Supplemental Figure 5, C-F). Together, the data indicate impaired glucose tolerance in $g l o 1^{-/-}$zebrafish; however, this had not yet translated into an obvious organ phenotype.

Obesity is a major risk factor for the development of type 2 diabetes mellitus, and we hypothesized that overfeeding of $\mathrm{glol}^{-{ }^{-}}$zebrafish could provoke a type 2 diabetes phenotype that leads to organ damage in $\mathrm{glol}^{-/-}$zebrafish. Therefore, a previously published model of diet-induced obesity, which includes an overfeeding period of 8 weeks with Artemia (43), was chosen for further experiments. Artemia consist of $22 \%$ fat, $16 \%$ carbohydrate, and $44 \%$ protein (dry weight basis) (44). To analyze the possible impact of Artemia feeding on dietary MG intake, dicarbonyl, AGEs, and oxidation adduct levels were determined by LC/MS in Artemia, hatched for 2 days. MG content of Artemia was about 5 to $10 \mathrm{pmol} / \mathrm{mg}$ tissue (Supplemental Figure 6), which is similar to mice (11) and zebrafish tissue MG levels (Figure 5B). At the end of the overfeeding period, the weight and fasting blood glucose level were measured in $\mathrm{glol}^{-/-}$zebrafish. Overfed fish gained significantly more weight than fish in the control-fed groups (Figure 6A) and overfeeding altered liver morphology that resulted in a fatty liver and increased accumulation of lipid droplets in the whole fish (Figure 6B). However, there was no difference in weight gain between $\mathrm{glol}^{+/+}$and $\mathrm{glo1}^{-/-}$zebrafish. Fasting blood glucose levels in the normal food intake $\mathrm{glo}^{1^{+/}}$and $\mathrm{glol}^{-/-}$zebrafish again had blood glucose levels of about 30 to $40 \mathrm{mg} / \mathrm{dL}$. Furthermore, overfed $\mathrm{glol}^{+/+}$zebrafish showed no significant increase in fasting blood glucose levels, which is in line with published data (42). Remarkably, overfed $\mathrm{glol}^{-1-}$ zebrafish showed an increased fasting blood glucose level of $51 \mathrm{mg} / \mathrm{dL}$ (Figure 6C). Together, the data suggest that Glo1 is dispensable for zebrafish development but makes $\mathrm{glol}^{-1-}$ animals susceptible to the development of diabetes-like conditions.

Overfeeding induces alterations of retinal vasculature in $\mathrm{glo1}^{-1-}$ zebrafish. Because overfeeding induced hyperglycemia in $g l o 1^{-1-}$ zebrafish, we addressed the question of whether this condition could lead to organ alterations in zebrafish and specifically focused on retinal blood vessels. In diabetic patients, hyperglycemia is associated with the development and progression of diabetic retinopathy (45). To identify retinal vascular alterations (Figure 7), we used a recently published protocol for analysis of the adult retinal vessel architecture (46). After overfeeding of $\mathrm{glol}^{-/-}$zebrafish, the retinae were dissected and analyzed by confocal microscopy (Figure 7C). The retinal vessels were not affected in $\mathrm{glol}^{-{ }^{-}}$zebrafish under normal feeding conditions (Figure 7, A, B, and D) which is in agreement with the trunk vasculature in $\mathrm{glo}^{\mathrm{I}^{-1}}$ larvae at $96 \mathrm{hpf}$ (Figure 2). However, we observed a significantly increased angiogenic sprout formation of the retinal vasculature in $g l o 1^{-/-}$overfed zebrafish (Figure 7, A, B, and D).

MG induces vascular alterations in zebrafish larvae (32); therefore, we determined MG levels in the eyes of the different animal groups. Under normal feeding conditions in fasted zebrafish, MG eye concentrations of $\mathrm{glol}^{-1-}$ zebrafish were not significantly increased (Figure 8A) and MG eye concentrations in fasted over- 
A

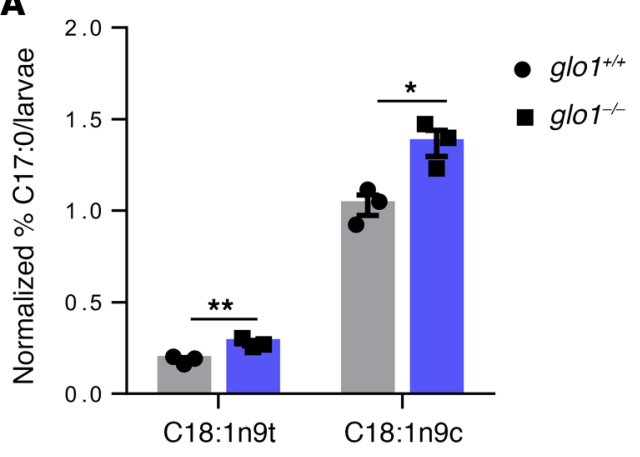

C

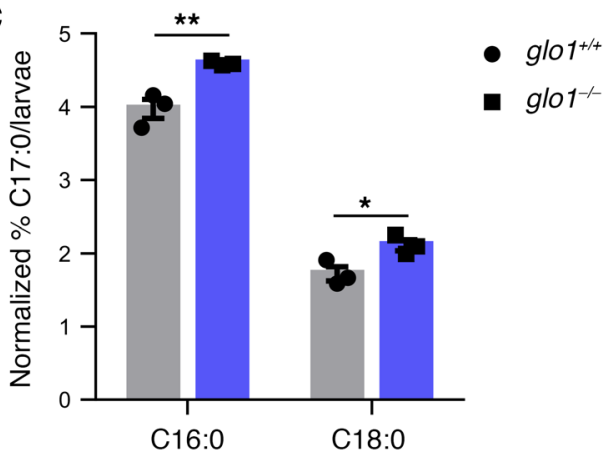

B

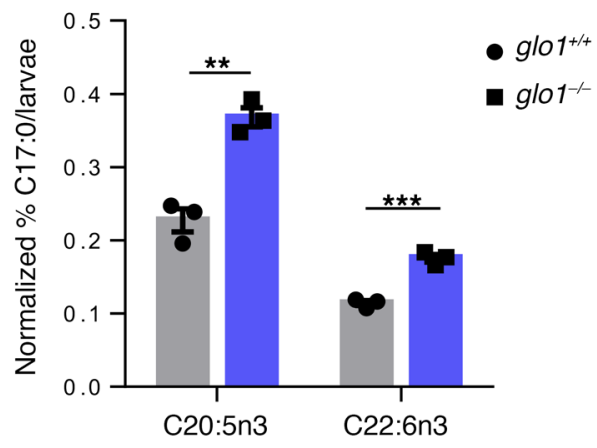

D

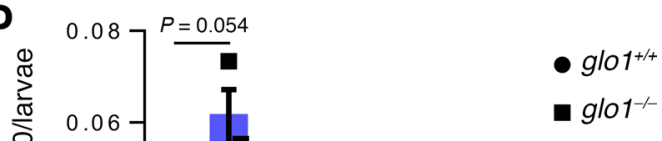

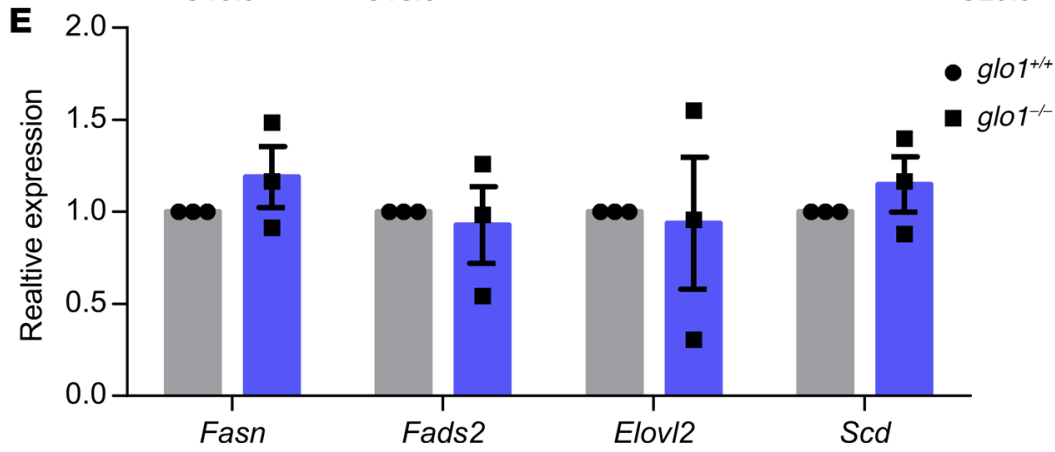

Figure 4. Altered fatty acid metabolism in glo1-/- zebrafish larvae. (A-D) Omega-3 (A), omega-9 fatty acids (B), and saturated fatty acids (C and D) levels are increased in $\mathrm{gl01^{-1- }}$ in comparison with $\mathrm{gl01^{+/+ }}$ zebrafish larvae at $96 \mathrm{hpf}$. Whole zebrafish larvae lysates at $96 \mathrm{hpf}$ were used for FAME-CC-MS analysis and results were normalized to percentage of [17:0, which was added before cell lysis; $n=3$ clutches with $40-50$ larvae; mean \pm SEM. (E) Expression of fatty acid synthesis genes is not altered in glo $1^{-1-}$ in comparison with $\mathrm{glo1^{+/+ }}$ larvae at $96 \mathrm{hpf}$. Expression of genes was determined by RT-qPCR and normalized to $\beta$ actin. Values for $\mathrm{glo1^{+/+ }}$ zebrafish embryos were standardized to $1, n=3$ clutches with 30-40 larvae; mean \pm SEM. For statistical analysis Student's $t$ test was applied. ${ }^{*} P<0.05$; ${ }^{* *} P<0.01$; ${ }^{* *} P<0.001$. Fasn, fatty acid synthase; Fads2, fatty acid desaturase 2; Elovl2, fatty acid elongase 2; Scd, stearoyl-CoA desaturase; ns, not significant; C18:1n9t, elaidic acid methyl ester; C18:1n9c, oleic acid methyl ester; C16:0, palmitic acid methyl ester; C18:0, stearic acid methyl ester; C20:0, arachidic acid methyl ester; C22:0, behenic acid methyl ester; C24:0, lignoceric acid methyl ester; C20:5n3, cis-5,8,11,14,17-eicosapentaenoic acid methyl ester; C22:6n3, cis-4,7,10,13,16,19-docosahexaenoic acid methyl ester.

fed $\mathrm{glol}^{+/+}$and $\mathrm{glo}^{\mathrm{I}^{-/}}$animals remained normal. In contrast, eye MG levels in postprandial $\mathrm{glol}^{\mathrm{I}^{-/}}$zebrafish strongly increased 4-fold (Figure 8B); however, postprandial eye MG concentrations in overfed glo $^{-1-}$ zebrafish returned to baseline levels. Taken together, these data show that overfed $\mathrm{glo}^{-/-}$zebrafish are susceptible to the development of hyperglycemia leading to retinal blood vessel alterations, which are most likely driven by hyperglycemia and not caused by increased MG levels.

Overfeeding leads to insulin resistance in the liver in zebrafish. Glo1-1- zebrafish had transiently increased postprandial blood glucose levels (Figure 5D) and overfeeding increased fasting blood glucose levels (Figure 6C), which led to vascular alterations in the retina (Figure 7, A, B, and D). To access the underlying mechanisms, activation of the p70-S6 kinase was determined as a downstream target of the insulin-signaling cascade. Increased activation of S6 kinase leads to the induction of insulin resistance through a negative feedback loop via blocking insulin receptor substrate 1 phosphorylation $(47,48)$. First, we analyzed S6 kinase phosphorylation in livers of $\mathrm{glol}^{+/+}$zebrafish after fasting and postprandi- 
A

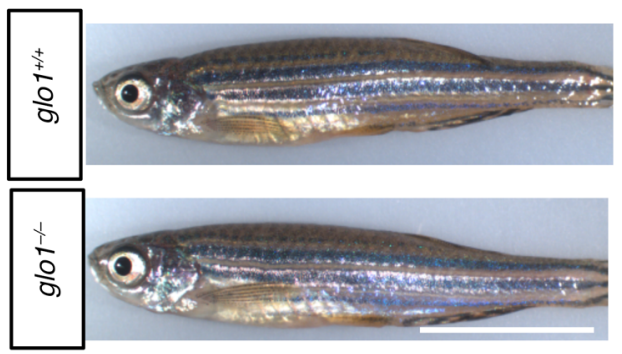

B

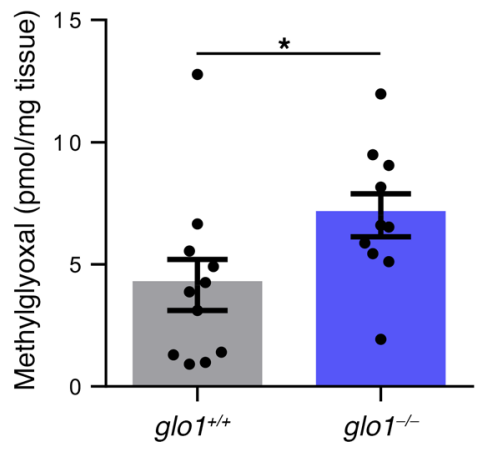

C

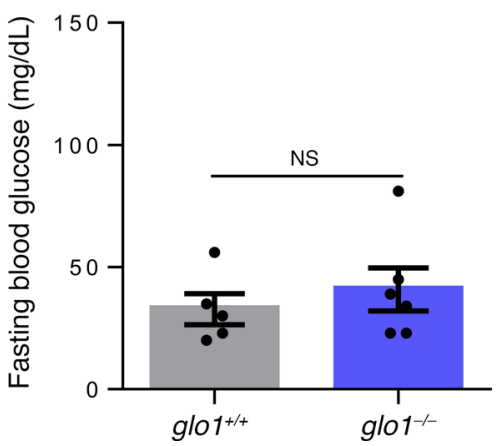

D

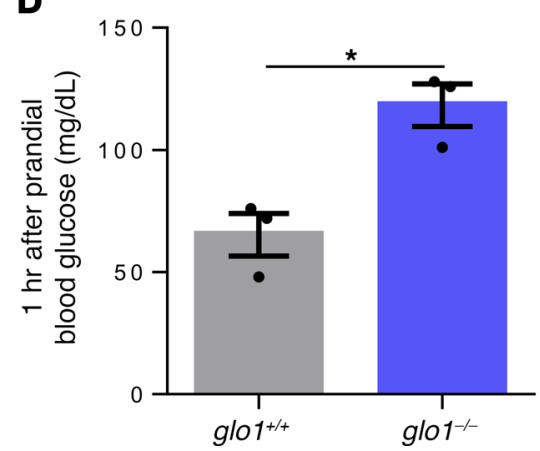

E

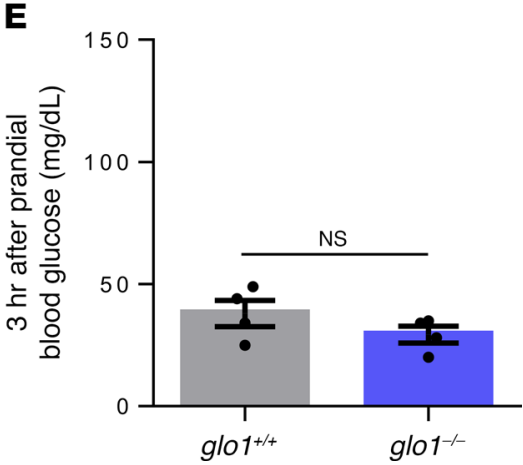

Figure 5. Altered MG detoxification and glucose metabolism in juvenile and adult glo1-/- zebrafish. (A) Light microscopic images of adult male glo1 $1^{-/-}$and $\mathrm{glo1^{+/+ }}$ zebrafish show normal gross morphology and no growth retardation of both groups. White scale bar: $5 \mathrm{~mm}$. (B) Methylglyoxal levels of whole body lysates are increased in $\mathrm{g} / 01^{-/-}$compared with $\mathrm{glo1^{+/+ }}$ juvenile zebrafish. Methylglyoxal was determined by LC-MS/MS in juvenile

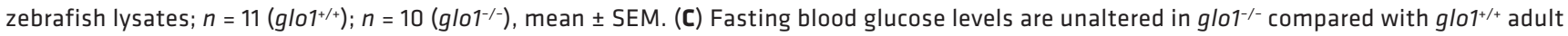
zebrafish; $n=5\left(\mathrm{glot}^{+/+}\right) ; n=6\left(\mathrm{~g} / 01^{-/-}\right)$, mean $\pm \mathrm{SE}$. (D) One hour after feeding, postprandial blood glucose levels are increased in $\mathrm{glo1^{-/ }}$ in comparison with $\mathrm{glo1^{+/+ }}$ adult zebrafish; $n=3\left(\mathrm{glo1^{+/+ }}\right) ; n=3\left(\mathrm{~g} / 0 \mathrm{1}^{-/-}\right)$, mean \pm SEM. (E) Three hours postprandial, blood glucose levels of $\mathrm{g} / 0 \mathrm{1}^{+/+}$and $\mathrm{glo1^{-/- }}$

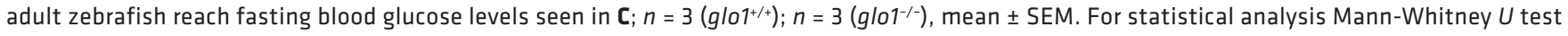
(B) and Student's $t$ test $(\mathbf{C}-\mathbf{E})$ were applied. ${ }^{*} P<0.05$. ns, not significant.

ally. The results showed a strong postprandial increase in phosphorylation of S6 kinase compared with a baseline phosphorylation after fasting, which proves an activation of S6 kinase after feeding (Figure 9, A and B) in zebrafish. Next, we compared S6 kinase activation in livers and skeletal muscles of postprandial $\mathrm{glo}^{\mathrm{I}^{++}}$and $\mathrm{glo1^{-/- }}$ animals and identified an increased S6 kinase activation in normal-fed glo1 $^{-/-}$livers compared with normal-fed $g l o 1^{+/+}$livers (Figure 9, C-F). This observation suggests an early feedback of the inhibition of the insulin signaling cascade through increased S6 kinase activation as a possible mechanism leading to transiently increased postprandial blood glucose levels in $\mathrm{glo1}^{-1-}$ zebrafish (Figure 5D) and again indicating impaired glucose tolerance in $g l o 1^{-/-}$zebrafish. In contrast with normal-fed zebrafish, overfeeding of $\mathrm{glol}^{+/+}$and $\mathrm{glol}^{-/-}$zebrafish completely abrogated postprandial S6 kinase activation in liver tissue (Figure 9, C and D). In skeletal muscle tissue of overfed $\mathrm{glo1}^{+/+}$ and $g l o 1^{-/-}$zebrafish, postprandial S6 kinase activation was reduced, but this reduction was not significant (Figure 9, E and F). Together, the data suggest that overfeeding of zebrafish blocks postprandial S6 kinase activation, but only in combination with Glo1 loss, leading to fasting hyperglycemia (Figure 6C) and consequently to retinal blood vessel alterations (Figure 7).

\section{Discussion}

This study established for the first time to our knowledge a Glo1 knockout in zebrafish and showed longterm effects of compromised MG metabolism affecting glucose metabolism and retinal blood vessels. Loss of Glo1 increases endogenous MG levels, induces impaired glucose tolerance in zebrafish indicated by elevated postprandial blood glucose levels and increased S6 kinase activation, and leads to fasting hyperglycemia and retinal vascular alterations under high nutrition intake (Figure 10).

The main conclusion of the study is that a knockout of glyoxalase 1 alone induces impaired glucose tolerance, but it is insufficient to induce vascular organ alterations. Although $\mathrm{glol}^{-/-}$zebrafish had no 
A

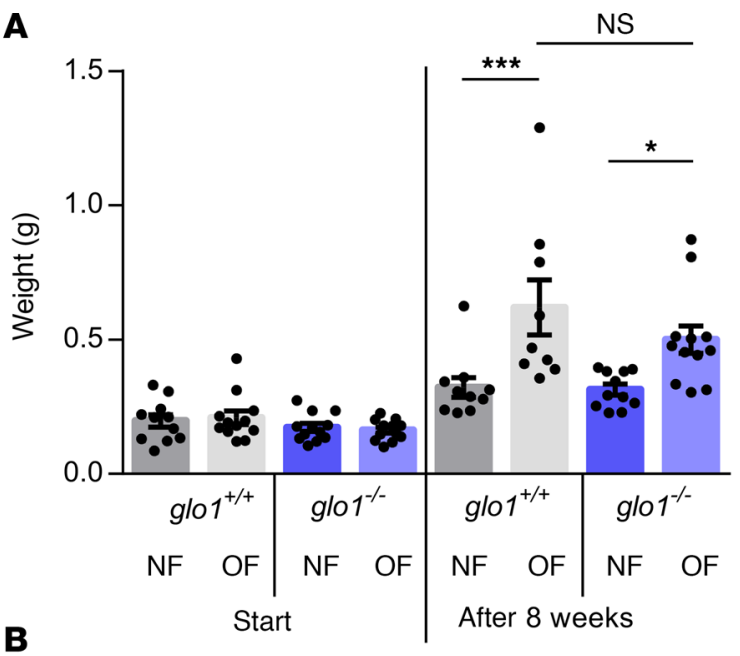

Normalfed liver
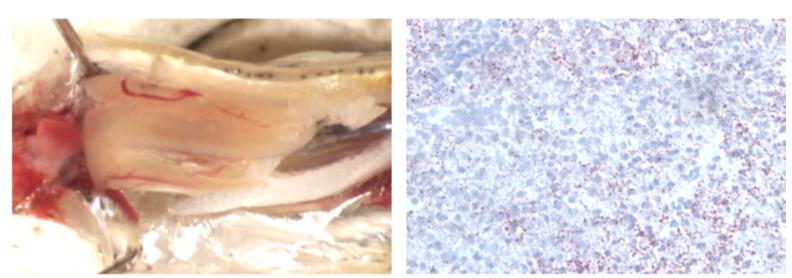

Overfed liver
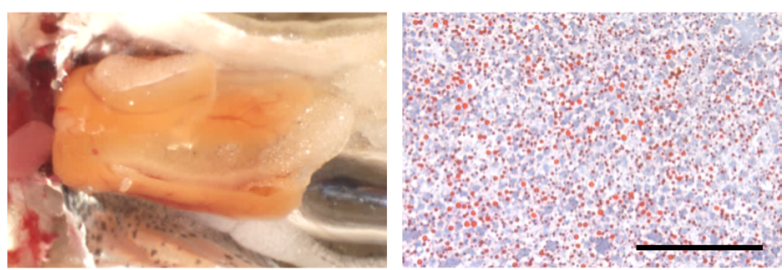

C

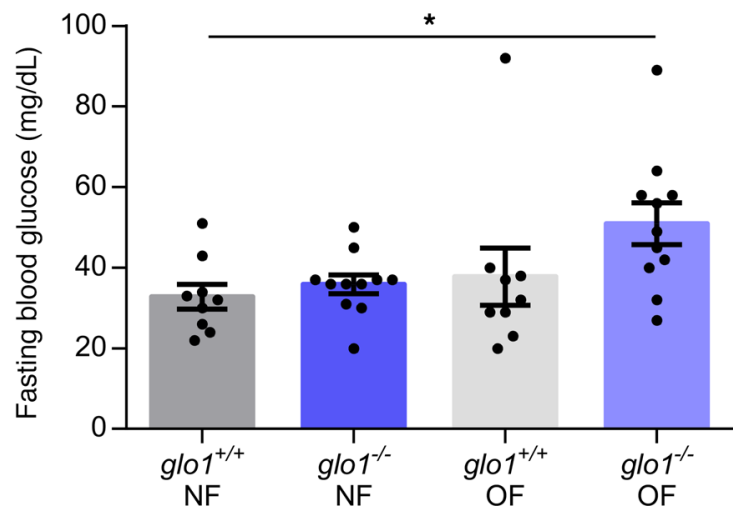

Figure 6. Diet-induced obese glo1 $^{-1-}$ zebrafish develop hyperglycemia. (A) After 8 weeks of overfeeding, zebrafish in the overfed group have gained significantly more weight than zebrafish in the normal-fed group, whereas there was no difference dependent on the glo1 genotype. Weight was measured at the start and at the end of the feeding period. Results were obtained from 3 independent experiments; $n=9$ to 12 fish per group; mean \pm SEM. (B) After the overfeeding period, livers of overfed fish show high fed content compared with livers of normal-fed fish and are surrounded by lipid droplets. Images were taken using a light microscope. Frozen liver sections were stained with Oil Red O. Black scale bar: $100 \mu \mathrm{m}$. (C) Fasting blood glucose levels are increased in glo1-/- overfed fish in comparison with $\mathrm{glo}^{+/+}$normal-fed fish; $n=$ 9 to 12 fish per group; mean \pm SEM. One-way ANOVA followed by Sidak's multiple-comparisons test was applied. ${ }^{*} P<0.05$; ${ }^{*} P<0.01$; ${ }^{* * *} P<0.001$. OF, overfed; NF, normal-fed; ns, not significant.

growth deficit, the metabolization of postprandial blood glucose was delayed in adult glo1 ${ }^{-1-}$ zebrafish, whereas fasting blood glucose was not altered. Using a zebrafish vascular reporter gene line, the increased MG levels could not be linked to alterations in the retinal vasculature. Thus, a second hit (e.g., diet-induced obesity), was necessary to induce permanent hyperglycemia in $\mathrm{glo}^{-{ }^{--}}$zebrafish, consequently leading to retinal vascular alterations. The data support the previous models for dicarbonyl stress being a cause for type 2 diabetes initiation (25), whereas in zebrafish a Glo1 knockout alone is not sufficient to progress to fasting hyperglycemia. Instead, $g l o 1^{-/-}$zebrafish can maintain euglycemia as long as they eat normally and fasting hyperglycemia develops only with the onset of diet-induced obesity. Whether other 

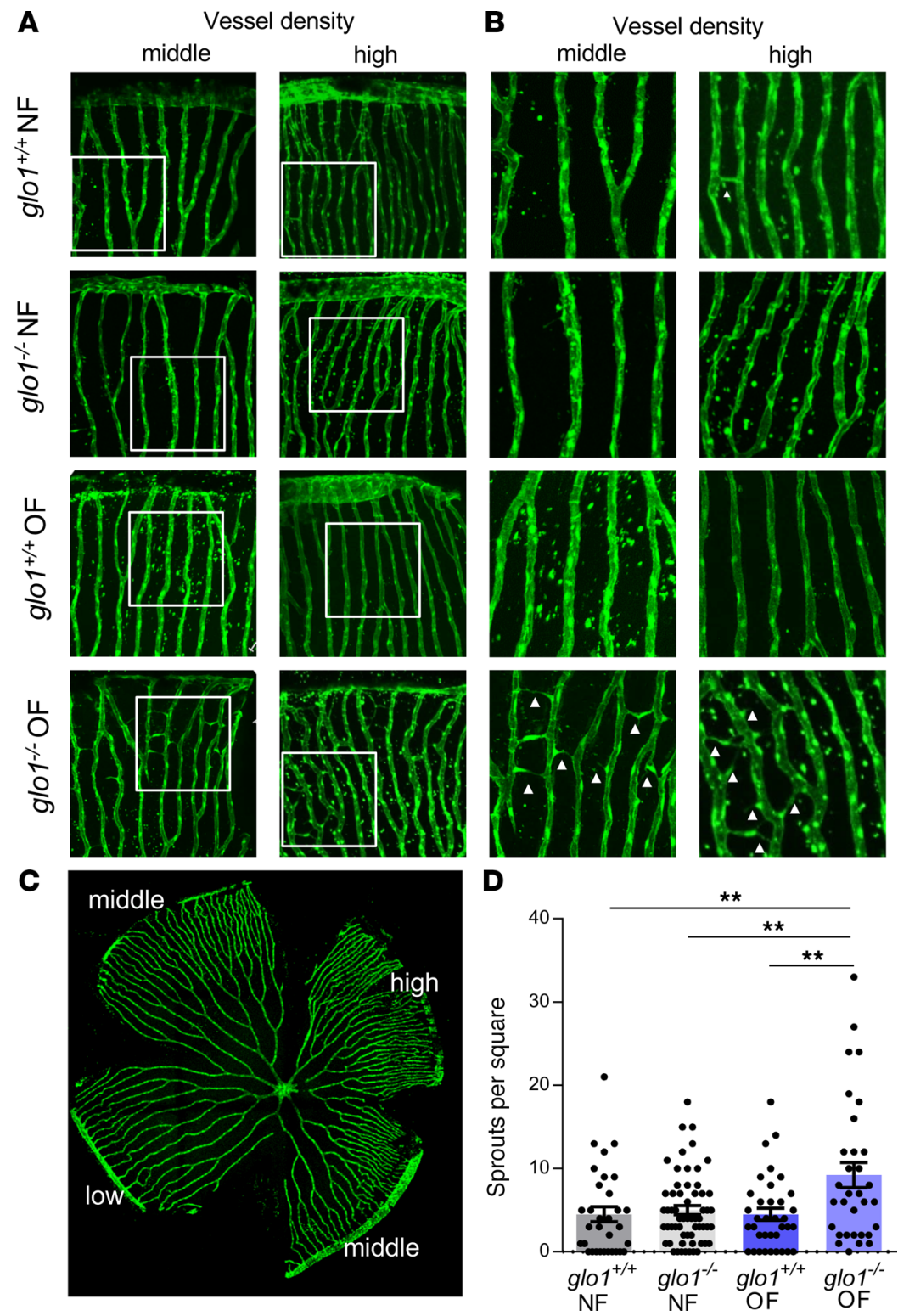

Figure 7. Increased retinal blood vessel sprouting in diet-induced obese glo1 $^{-/-}$zebrafish. (A and B) Confocal images of TG(fli:EGFP) retinas of adult zebrafish show increased sprout formation in the retina of $\mathrm{glo1^{-1 }}$ overfed zebrafish. The images represent 350- $\mu \mathrm{m}$ squares that were cut out of 2 different parts of the retina, containing areas with high and middle vessel density. White arrowheads indicate vessel sprouts and white boxes (A) indicate region seen in $\mathbf{B}$. (C) Confocal image of a whole retina showing different areas of vessel density. (D) Quantification of vessel sprouts; $n=4$ to 7 zebrafish per group, 32 to 60 squares per group were analyzed. For statistical analysis 1-way ANOVA followed by Sidak's multiple-comparisons test was applied. ${ }^{* *} P<0.01$. OF, overfed; NF, normal-fed.

factors beyond high calorie intake, such as altered activities for enzymes linked for example to glyoxalase metabolism, such as Aldh and Akr enzymes, may lead to fasting hyperglycemia in glo $^{-1-}$ zebrafish is $^{-1}$ currently unknown but will be investigated in future studies.

In this study, we identified an increased phosphorylation of the p70-S6 kinase on Thr389 in liver and skeletal muscle tissue of normal-fed glo $^{-1-}$ zebrafish. It was previously shown that S6 kinase directly inhibits insulin receptor substrate 1 through a negative feedback loop indicated by phosphorylation of serine residues and an S6 kinase knockout protects against diet-induced insulin resistance (47-49). These data suggest an increased S6 kinase phosphorylation as an early and transient mechanism responsible for postprandial elevated blood glucose levels in normal-fed glo $^{-/-}$zebrafish. Using a diet-induced obesity model for $g l o 1^{+/+}$and $\mathrm{glol}^{-/-}$mutants, which shares pathophysiological pathways 

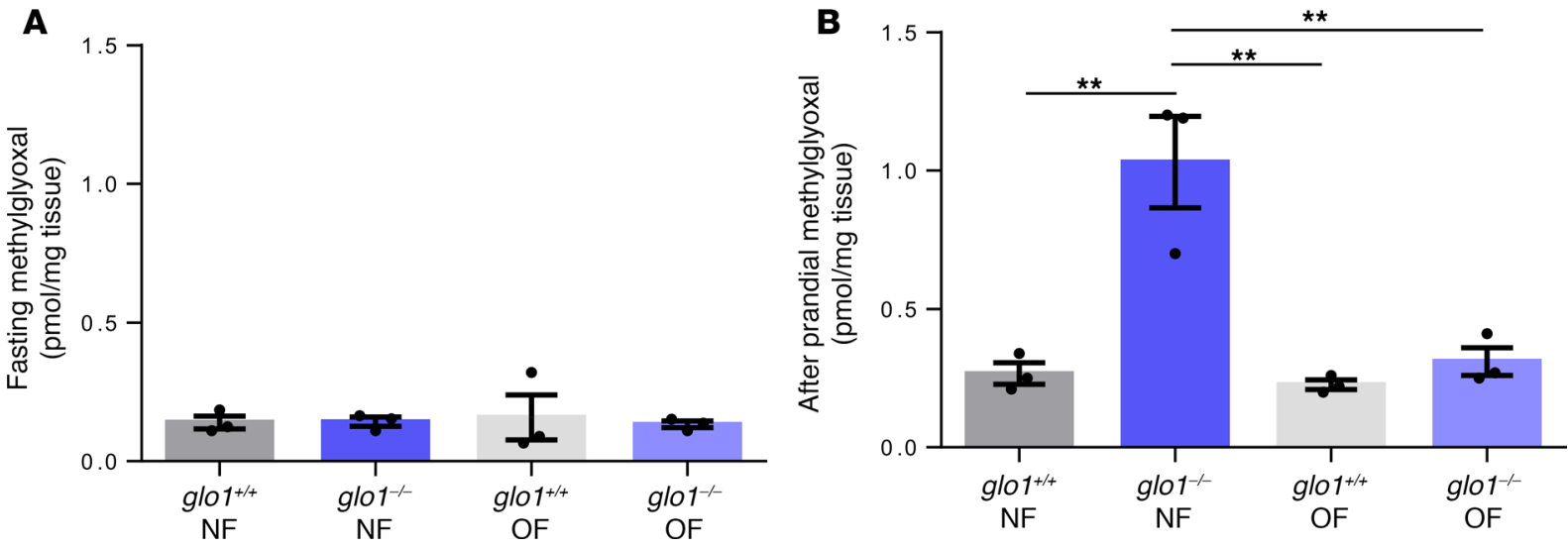

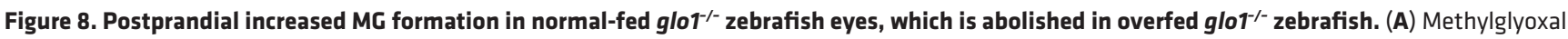
concentrations in zebrafish eyes are not significantly altered in overfed and normal-fed $\mathrm{g} / 01^{-1-}$ in the fasting state compared with overfed and normal-fed

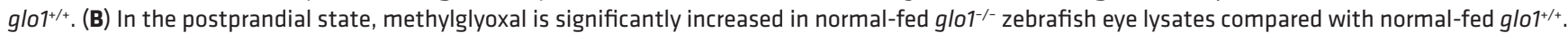
After overfeeding, postprandial methylglyoxal in the eye of $\mathrm{glo1^{-/- }}$ zebrafish is significantly decreased compared with normal-fed $\mathrm{glo1^{-/- }}$ zebrafish. Methylglyoxal was determined by LC-MS/MS in zebrafish eye lysates after 16 hours fasting or 1 hour after feeding; $n=3$ fish per group; mean \pm SEM. For statistical analysis, 1-way ANOVA followed by Tukey's multiple-comparisons test was applied. ${ }^{* *} P<0.01$. OF, overfed; NF, normal-fed.

with mammalian obesity (43), we found a total inhibition of S6 kinase phosphorylation in livers in both genotypes, indicating inhibition of the insulin receptor pathway in livers. Surprisingly, fasting blood glucose levels were not increased in overfed $\mathrm{glol}^{+/+}$zebrafish. Yet, this observation agrees with a recently published study showing that skeletal muscle insulin resistance zebrafish maintained normal fasting blood glucose levels over a long period of time by an increase in beta cell mass formation. Nevertheless, overfeeding also induced progression to hyperglycemia in this zebrafish model of skeletal muscle insulin resistance (50). Thus, the missing hyperglycemia in overfed $\mathrm{glol}^{+/+}$zebrafish implies increased insulin secretion or use of an alternative glucose metabolizing pathway, such as the polyol pathway. The data may also indicate that the failure of $g l o 1^{-1-}$ zebrafish to increase insulin is the result of dysfunctional beta cells, but this still needs to be determined.

Unexpectedly, glo1 ${ }^{-1-}$ zebrafish showed only a 1.5-fold increase in MG. Apart from triose intermediates of glycolysis, MG is formed by lipid, threonine, and acetone metabolism (51). To avoid the accumulation of MG, the cell has established rescue mechanisms. In addition to detoxifying enzyme systems, a study in drosophila recently suggested an upregulation of the fatty acid synthase as a mechanism to avoid high dicarbonyl tissue levels (52). In zebrafish, both mechanisms, the upregulation of Aldh activity and the increased formation of fatty acids in zebrafish larvae, could partially compensate and maintain MG levels in a range that is only 50\% higher than in wild-type animals. These results highlight variability in compensation capacity of established animal models, like zebrafish, flies, and mice to counterbalance for loss of Glo1. Whereas mice effectively prevent increased formation of $\mathrm{MG}$ (11), zebrafish and flies exhibit a 50\% increase in glo1-knockout animals (25). However, it must also be noted that glo1 $^{-1-}$ zebrafish eyes showed a postprandial, but not fasting, 4-fold increase in MG, implying a limited capacity of alternative rescue mechanisms. Interestingly, this transient MG increase was insufficient to alter retinal blood vessels. Unexpectedly, increased MG levels in the eye of postprandial normal-fed $\mathrm{glol}^{-1-}$ zebrafish were completely abolished in overfed $\mathrm{glol}^{-/-}$zebrafish. This finding suggests overfeeding results in the development of unknown metabolic alterations to avoid accumulation of

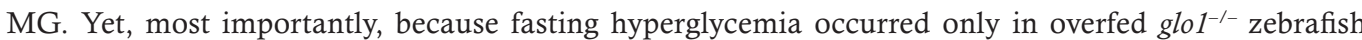
and retinal vascular alterations developed only in overfed $g l o 1^{-1-}$ zebrafish, the data suggest glucose, but not $\mathrm{MG}$, as the damaging factor in retinal blood vessels in $\mathrm{glol}^{-1-}$ zebrafish.

In conclusion, the findings of this study in zebrafish identified a Glo1 knockout, accompanied by an increase of MG levels, as a driver of insulin resistance leading to hyperglycemia and retinal blood vessel alterations under high nutrient intake only. As long as MG is effectively detoxified, there is no harm to the organism, but with the failure of detoxification, the increase in MG, together with diets or other metabolic alterations, contributes to the initiation and progression of insulin resistance and organ alterations. 
A

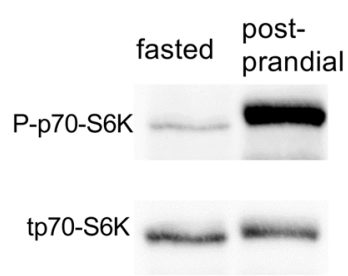

C

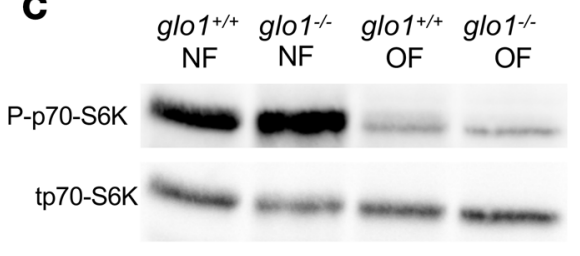

$\mathbf{E}$

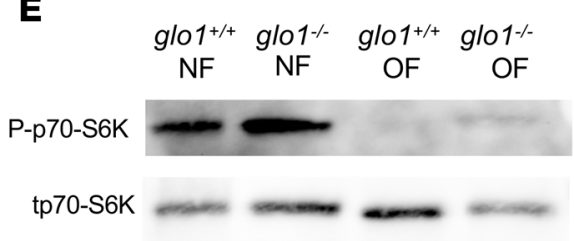

B

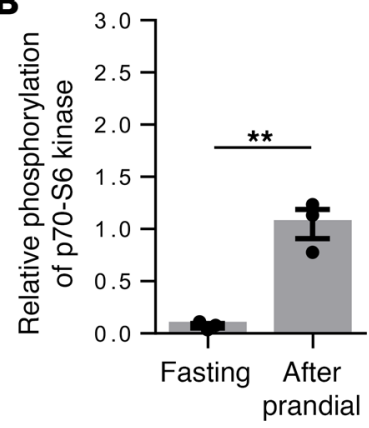

D

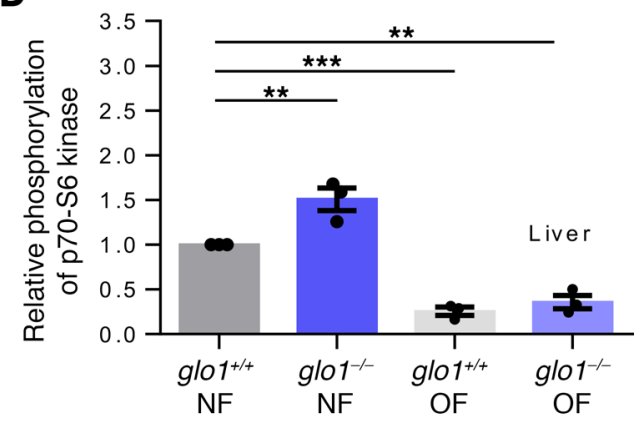

$\mathbf{F}$

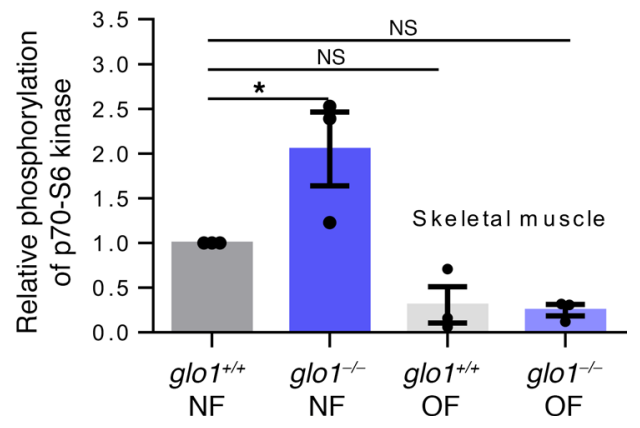

Figure 9. Insulin resistance in livers of overfed zebrafish. (A) Representative Western blot shows postprandial induction of p70-S6 kinase phosphorylation in livers of $\mathrm{glo}^{1^{++}}$zebrafish. Total (t) p70-S6 kinase (S6K) serves as loading control. (B) Quantification of p70-S6 kinase phosphorylation in liver lysates of $\mathrm{glo}^{1^{+/+}}$zebrafish in fasted and postprandial state; $n=3$, mean \pm SEM. (C and $\mathbf{E}$ ) Representative Western blots shows increased postprandial phosphory-

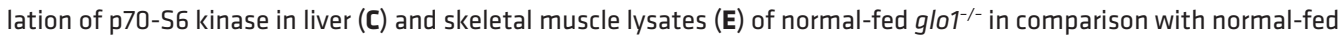
$\mathrm{glO}^{+/+}$zebrafish. Overfed $\mathrm{glo1^{+/+ }}$ and $\mathrm{glo1^{-/ }}$ zebrafish livers (C) and skeletal muscle tissue (E) showed strongly reduced postprandial phosphorylation of p70-S6 kinase in comparison with normal-fed $g / 01^{+/+}$zebrafish. Total p70-S6 kinase serves as loading control. ( $D$ and $\mathbf{F}$ ) Quantification of p70-S6 kinase phosphorylation in liver and skeletal muscle tissue of normal-fed and overfed $\mathrm{glo}^{+/+}$and $\mathrm{glo1^{-/- }}$ zebrafish; $n=3$, mean \pm SEM. For Western blot quantification Image/ was used. For statistical analysis, Student's $t$ test (B) and 1-way ANOVA followed by Tukey's multiple-comparisons test (D and F) were applied. ${ }^{*} P<0.05 ;{ }^{*} P<0.01 ;{ }^{* *} P<0.001$. OF, overfed; NF, normal-fed; ns, not significant.

\section{Methods}

Zebrafish husbandry and zebrafish lines. Embryos of AB wild-type, $\operatorname{Tg}(f l i 1: E G F P)(53)$, and $\operatorname{Tg}(w t 1 b: E G F P)(54)$ were raised and staged as described according to hours after fertilization (i.e., hpf) (55). Embryos/larvae were kept in egg water at $28.5^{\circ} \mathrm{C}$ with $0.003 \%$ PTU to suppress pigmentation. Adult zebrafish were kept under 13 -hour light/11-hour dark cycle and fed with freshly hatched Artemia and fish flake food. Between 30 and 89 days after fertilization, fish are referred to as juvenile and from 90 days and greater after fertilization as adult fish.

Antibodies and reagents. Antibodies and reagents used included Proteinase K $(10 \mathrm{mg} / \mathrm{mL}$ stock, Roche Recombinant PCR grade), MG solution 6.49 M (Sigma-Aldrich), rat anti-Glo1 (6F10, Abcam), rabbit antiphospho-p70-S6 kinase (Thr389, 108D2, Cell Signaling Technology), rabbit anti-p70-S6 kinase (49D7, Cell Signaling Technology), goat anti-actin (Santa Cruz Biotechnology), and horseradish peroxidase-conjugated antibodies rabbit anti-mouse, rabbit anti-rat, goat anti-rabbit, and rabbit anti-goat (Dako). 


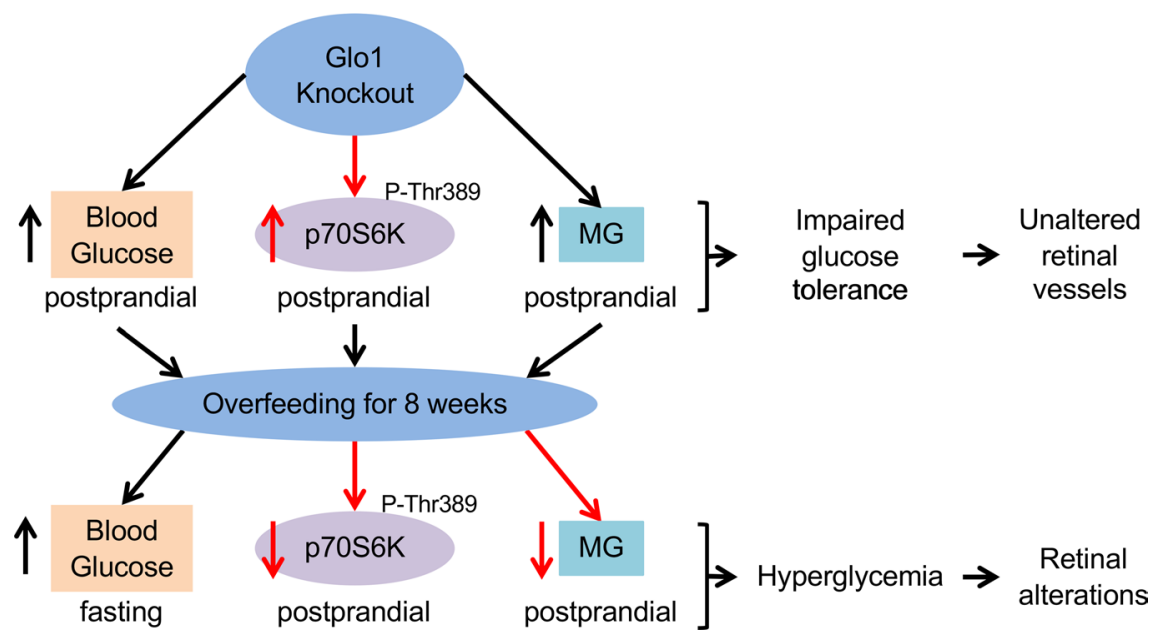

Figure 10. Model for the role of the glyoxalase 1 in zebrafish. Knockout of glyoxalase 1 in zebrafish leads to impaired glucose tolerance indicated by an increase of postprandial blood glucose, increase of postprandial S6 kinase phosphorylation, and increase of postprandial MG levels. Overfeeding of $\mathrm{glo1^{-/ }}$ zebrafish induced progression of postprandial elevated blood glucose levels to fasting elevated blood glucose levels, resulting in the formation of altered retinal blood vessels. This progress is accompanied by decreased postprandial 56 kinase phosphorylation and MG levels.

Mutant generation. One guide RNA targeting Exon 3 of glo1 was designed (ZiFiT-Targeter 3.3) and cloned into a T7-driven promotor expression vector (pT7-gRNA, Addgene). glo1-CRISPR-for: 5'-TAGGCTCTACGGGAGAACGTCC-3'， glo1-CRISPR-rev: 5'-AAACGGACGTTCTCCCGT AGAG-3'. Cas 9 mRNA was achieved by in vitro transcription of pT3TS-nCas9n vector (Addgene). Synthesis of mRNA was performed using the T7 mMessage mMachine Kit and T3 MEGAshortscript Kit following the protocol of the manufacturer (Invitrogen). For mutant generation, $1 \mathrm{~nL}$ of a mixture containing 200 $\mathrm{pg} / \mathrm{nL}$ guide RNA and $200 \mathrm{pg} / \mathrm{nL}$ Cas9-mRNA were injected into the cell of an AB wild-type embryo of the 1-cell stage (56). For generation of heterozygous mutants for glo1, F0 fish were analyzed for germline transmission, crossed with $T g(f l i 1: E G F P)$ and $T g(w t 1 b: E G F P)$ zebrafish and raised (57). Genotyping was performed by Sanger sequencing of PCR-products which were generated using gene specific primers which bind to flanking regions next to the target sequence in Exon 3 of glo1. glo1-genotyping-for: 5'-TAAACGTGCACCCTGAACTG-3', glo1-genotyping-rev: 5'-CCCAACACTAGCACACACAC-3'. Mutations were analyzed using yosttools (58).

$M G, 3-D G$, and glyoxal measurements. MG, 3-DG, and glyoxal levels of zebrafish larvae, whole body lysates, and eyes were determined by LC-MS/MS, as described previously (59-61). Tissue MG levels were determined by derivatization with 1,2-diaminobenzene (DB). Briefly, pre-weighed amounts of tissue (ca.10 mg) were homogenized in ice-cold $20 \%$ (wt/vol) trichloroacetic acid in $0.9 \%$ (wt/vol) sodium chloride $(20 \mu \mathrm{L})$ and water $(80 \mu \mathrm{L})$. An aliquot $(5 \mu \mathrm{L})$ of the internal standard (13C3-MG; $400 \mathrm{nM})$ was then added and the samples vortexed mixed. Following centrifugation $\left(20,817 \mathrm{~g} ; 5\right.$ minutes at $\left.4^{\circ} \mathrm{C}\right), 35$ $\mu \mathrm{L}$ of the supernatant was transferred to an HPLC vials containing a $200-\mu \mathrm{L}$ glass interest. An aliquot $(5 \mu \mathrm{L})$ of $3 \%$ sodium azide (wt/vol) was then added to each sample followed by $10 \mu \mathrm{L}$ of $0.5 \mathrm{mM} \mathrm{DB}$ in $200 \mathrm{mM} \mathrm{HCl}$ containing $0.5 \mathrm{mM}$ diethylenetriaminepentaacetic acid (DETAPAC) in water. The samples were then incubated for 4 hours at room temperature, protected from the light. Samples were then analyzed by LC-MS/MS using an ACQUITY ultra-high-performance liquid chromatography system with a Xevo-TQS LC-MS/MS mass spectrometer (Waters). The column was a Waters BEH C18 $(100 \times 2.1$ $\mathrm{mm})$ and guard column $(5 \times 2.1 \mathrm{~mm})$. The mobile phase was $0.1 \%$ formic acid in water with a linear gradient of $0 \%$ to $100 \% 0.1 \%$ formic acid in $50 \%$ acetonitrile/water over 0 to 10 minutes; the flow rate was $0.2 \mathrm{~mL} / \mathrm{min}$ and column temperature was $5^{\circ} \mathrm{C}$. The capillary voltage was $0.5 \mathrm{kV}$, the cone voltage $20 \mathrm{~V}$, the interscan delay time $100 \mathrm{~ms}$, the source and desolvation gas temperatures $150^{\circ} \mathrm{C}$ and $350^{\circ} \mathrm{C}$, respectively, and the cone gas and desolvation gas flows were 150 and $800 \mathrm{~L} / \mathrm{h}$, respectively. Mass transitions (parent ion $\rightarrow$ fragment ion; collision energy), retention time, limit of detection, and recoveries were as follows: $145.0 \rightarrow 77.1,24 \mathrm{eV}, 5.93$ minutes, $0.52 \mathrm{pmol}$, and $98 \%$. 
Glycogen assay. Glycogen content of liver tissue(s) was measured according to manufacturer instructions of Glycogen Assay Kit (Biovision) and normalized to total protein content, as determined by the Bradford assay.

Lactate assay. Lactate content of liver tissue(s) was measured using an endpoint, enzymatic assay with L-lactate dehydrogenase, as described previously (62) and normalized to total protein content, as determined by the Bradford assay.

Protein-bound AGEs. The AGE content of liver tissue(s) and Artemia was determined by exhaustive enzymatic hydrolysis and LC-MS/MS, as described previously (63).

Incubation with $M G$. Approximately 80 fertilized eggs were incubated in a $10-\mathrm{cm}$ petri dish with $30-\mathrm{mL}$ solutions that were changed daily. Solutions contained egg water, $500 \mu \mathrm{M} \mathrm{MG}$, and 0.2\% 1-phenyl-2-thiourea.

Microscopy and quantification of vascular defects. For in vivo imaging, Tg(fli1:EGFP) embryos were anesthetized with $0.003 \%$ tricaine and embedded in 1\% low-melting-point agarose dissolved in egg water. Confocal images were performed using DM6000 B confocal microscope with Leica TCS SP5 DS scanner. Images were taken with $600 \mathrm{~Hz}, 1024 \times 512$ pixels, and $Z$-stacks were recorded with 1 - $\mu$ m thickness. For quantification of altered blood vessels, an inverted microscope (Leica DMI 6000 B) with a camera (Leica DFC420 C) and the Leica LAS application suite 3.8 software was used. The first 14 ISV pairs of each zebrafish larvae were analyzed for hyperbranching blood vessels at $96 \mathrm{hpf}$. Results are given as mean number of hyperbranches per larvae at $96 \mathrm{hpf}$.

Enzyme activity assays. Glo1 activity was determined spectrophotometrically monitoring the change in absorbance at $235 \mathrm{~nm}$ caused by the formation of S-D-lactoylglutathione. The assay mixture contained $2 \mathrm{mM}$ MG and $2 \mathrm{mM} \mathrm{GSH}$ in sodium phosphate buffer $\left(50 \mathrm{mM}[\mathrm{pH} 6.6], 37^{\circ} \mathrm{C}\right)$ and was incubated for 15 minutes to guarantee the complete formation of HTA. After the addition of the cytosolic protein fraction $(1 \mathrm{~g} / \mathrm{L})$, the change in absorbance was monitored for 15 minutes. The activity of Glo1 is described in units, where 1 unit is the amount of Glo1 that catalyzes the formation of $1 \mathrm{~mol}$ of $S$-D-lactoylglutathione/min (64).

For Akr and Aldh activity the change in absorbance was monitored by following the loss of NADPH or the rate of NADPH formation at $340 \mathrm{~nm}(10,65,66)$. Briefly, activity of $\mathrm{Akr}$ was determined in $0.1 \mathrm{M}$ sodium phosphate buffer ( $\mathrm{pH} 7.2)$ containing MG $(0.1-2 \mathrm{mM})$ and $0.1 \mathrm{mM} \mathrm{NADPH}$ at $25^{\circ} \mathrm{C}$. Aldh activity was assayed at $25^{\circ} \mathrm{C}$ in $75 \mathrm{mM}$ Tris- $\mathrm{HCl}$ (pH 7.6) containing $10 \mathrm{mM}$ BSA, $0.5 \mathrm{mM}$ NADP, and 0.1-2 mM MG.

Metabolite analysis by GC/MS and UPLC-FLR. Detection was done in cooperation with the Metabolomics Core Technology Platform from the Centre of Organismal Studies Heidelberg. At 96 hpf, zebrafish larvae were anesthetized with $0.003 \%$ tricaine, collected, and snap frozen in liquid nitrogen. Metabolites were determined via semi-targeted gas chromatography-mass spectrometry (GC/MS) analysis and ultra-performance liquid chromatography with fluorescence detection (UPLC-FLR) analysis (67). Frozen ground sample material from zebrafish was extracted in $360 \mu \mathrm{L}$ of $100 \% \mathrm{MeOH}$ for 15 minutes at $70^{\circ} \mathrm{C}$ with vigorous shaking. As internal standards, $20 \mu \mathrm{L}$ ribitol $(0.2 \mathrm{mg} / \mathrm{mL})$ and $10 \mu \mathrm{L}$ heptadecanoic acid $(0.2 \mathrm{mg} / \mathrm{mL})$ were added to each sample. After the addition of $200 \mu \mathrm{L}$ chloroform, samples were shaken at $37^{\circ} \mathrm{C}$ for 5 minutes. To separate polar and organic phases, $400 \mu \mathrm{L}$ water were added and samples were centrifuged for 10 minutes at 11,000 $\times g$. For the derivatization, $700 \mu \mathrm{L}$ of the polar (upper) phase were transferred to a fresh tube and dried in a speed-vac (vacuum concentrator) without heating. Pellets of the aqueous phase after extraction were re-dissolved in $20 \mu \mathrm{L}$ methoximation reagent containing $20 \mathrm{mg} / \mathrm{mL}$ methoxyamine hydrochloride (Sigma-Aldrich 226904) in pyridine (Sigma-Aldrich 270970) and incubated for 2 hours at $37^{\circ} \mathrm{C}$ with shaking. For silylation, $32.2 \mu \mathrm{L} N$-methyl- $N$-(trimethylsilyl)trifluoroacetamide (MSTFA; Sigma M7891) and $2.8 \mu \mathrm{L}$ Alkane Standard Mixture (50 mg/mL C $\mathrm{C}_{10}-\mathrm{C}_{40}$; Fluka 68281) were added to each sample. After incubation for 30 minutes at $37^{\circ} \mathrm{C}$, samples were transferred to glass vials for GC/MS analysis. To analyze total fatty acids, $150 \mu \mathrm{L}$ of the lower organic phase (chloroform) after extraction were transferred to a fresh $1.5-\mathrm{mL}$ reaction tube and dried in a speed-vac without heating. For transmethylation reactions, pellets were re-dissolved in $40 \mu \mathrm{L}$ TBME (tert-butyl methyl ether, Sigma-Aldrich) and $20 \mu \mathrm{L} \mathrm{TMSH}$ (trimethylsulfoniumhydroxid, Sigma-Aldrich), incubated for 45 minutes at $50^{\circ} \mathrm{C}$, and transferred to glass vials for GC/MS analysis of the fatty acid methyl esters (FAMEs). A GC/MS-QP2010 Plus (Shimadzu) fitted with a Zebron ZB 5MS column (Phenomenex; 30 meter $\times 0.25 \mathrm{~mm} \times 0.25 \mu \mathrm{m})$ was used for GC/MS analysis. The GC was operated with an injection temperature of $230^{\circ} \mathrm{C}$ and a $1-\mu \mathrm{L}$ sample was injected with split mode (1:10). The GC temperature program started with a 1-minute hold at $40^{\circ} \mathrm{C}$ followed by a $6^{\circ} \mathrm{C} / \mathrm{min}$ ramp to $210^{\circ} \mathrm{C}$, a $20^{\circ} \mathrm{C} / \mathrm{min}$ ramp to $330^{\circ} \mathrm{C}$, and a bake-out for 5 minutes at $330^{\circ} \mathrm{C}$ using Helium as carrier gas with constant linear velocity. The MS was operated with ion source and interface temperatures of $250^{\circ} \mathrm{C}$, a solvent cut time of 7 minutes, and a scan range $(\mathrm{m} / \mathrm{z}$ ) of 40 to 700 with an event time of $0.2 \mathrm{~s}$. The "GCMS solution" software (Shimadzu) was used for data processing. 
Reverse-transcription quantitative polymerase chain reaction analysis. Expression of fatty acid synthesis genes was analyzed using reverse-transcription quantitative polymerase chain reaction (RT-qPCR). Total RNA was isolated from TG(fli1:EGFP) zebrafish larvae at $96 \mathrm{hpf}$ using the RNeasy Mini Kit following the manufacturer's protocol (Qiagen). First-strand cDNA was generated from $1 \mu \mathrm{g}$ RNA using random hexamer primer and the Superscript II kit (Invitrogen). Primer design for zebrafish was done using NCBI or by Roche Universal Probe Library Assay Design Center and primers are listed in Supplemental Table 1. Gene expression was normalized to $\beta$-actin and values for $\mathrm{glo}^{+/+}$zebrafish embryos were standardized to 1 . qPCR was performed with the Light-Cycler 480 (Roche Diagnostics). For detection, SensiFAST SYBR No-Rox Kit (Bioline) or $2 \times$ sentiFAST probe No-ROX mix (Bioline) was used in 96-well reaction plates (Axon). For SYBR Green, detection signals of amplified products were verified using melting curve analysis. The RT-qPCR was repeated 3 times per group.

Blood glucose measurement. Fish were transferred in single boxes and fasted overnight. After 16 to 18 hours, fish were either directly euthanized for fasting experiments or fed with $0.5 \mathrm{~g}$ flake food for 1 hour followed by 1 or 3 hours of fasting for postprandial experiments. Afterwards, fish were euthanized in 250 $\mathrm{mg} / \mathrm{L}$ tricaine and blood glucose was measured according to Zang et al. (68).

Overfeeding of adult zebrafish as a diet-induced obesity model. Adult zebrafish were separated into 2 groups. The control group was fed once per day with freshly hatched Artemia (5 mg artemia cysts per fish) whereas the overfeeding group was fed 3 times per day with Artemia (in total $60 \mathrm{mg}$ cysts per fish) according to Oka et al. (43). Because of our usual feeding routine, we modified the protocol resulting in an additional time feeding with flake food of both groups. Fish were fed according to the protocol for at least 8 weeks. To evaluate weight gain, fish were weighed at the start and at the end of the overfeeding period. In addition, Oil Red O staining was performed to address liver fatty acid content. Therefore, fish were euthanized in $250 \mathrm{mg} / \mathrm{L}$ tricaine, livers were embedded in Tissue-tek, frozen using liquid nitrogen cooled isopentane, and cut using a cryostat (8- $\mu \mathrm{m}$ sections). After drying for 15 minutes, the sections were fixed in 4\% PFA/PBS for 15 minutes, rinsed with distilled water, and stained in $0.3 \%$ Oil Red O working solution (60\% isopropanol). After staining, sections were rinsed in distilled water and counterstained using Myer's hematoxylin. Images were taken using an inverted microscope (Leica DMI 6000 B) with a camera (Leica DFC420 C) and the Leica LAS application suite 3.8 software.

Preparation, microscopy, and quantification of retinal vasculature. Fish were euthanized in $250 \mathrm{mg} / \mathrm{L}$ tricaine, eyes were fixed in 4\% PFA/PBS overnight, and retinal vasculature was dissected and analyzed (46). Confocal images were performed using DM6000 B confocal microscope with Leica TCS SP5 DS scanner. Images were taken with $600 \mathrm{~Hz}, 1024 \times 1024$ pixels (retina vessels) and Z-stacks were 1.5 - $\mu \mathrm{m}$ thick. Quantification of sprouting vessels was performed in squares of $350 \mu \mathrm{m}$ generated using GIMP2 (https://www.gimp.org/) and ImageJ (NIH). Whole retinae were divided into 3 different areas according to their vessel density: low (25\%), middle $(50 \%)$, and high density (25\%). Within these areas, new blood vessels without a lumen formed by neoangiogenesis were counted as sprouts. The quantification was adjusted to the lowest number of obtained squares per area at a ratio 1:2:1 (low/middle/high density areas) using random.org sequence generator.

Western blot analysis. For analysis of Glo1 expression, zebrafish embryos were collected at $72 \mathrm{hpf}$, and Western blot was performed as described previously (69). For analysis of S6 kinase phosphorylation in liver and skeletal muscle extracts, fish were euthanized in $250 \mathrm{mg} / \mathrm{L}$ tricaine, and liver and skeletal muscle were dissected and snap frozen. Shortly before usage, proteins were extracted using the TissueLyser II (Qiagen).

Statistics. Statistical significance between different groups was analyzed using 2-sided unpaired Student's $t$ test, Mann-Whitney $U$ test, or 1 -way ANOVA as indicated using GraphPad Prism. $P$ values $<0.05$ were considered significant: ${ }^{*} P<0.05 ;{ }^{* *} P<0.01,{ }^{* * *} P<0.001$.

Study approval. All experimental procedures on animals were approved by the local government authority, Regierungspräsidium Karlsruhe (license no.: G-160/14) and carried out in accordance with the approved guidelines.

\section{Author contributions}

EL performed experiments, analyzed data, and wrote the manuscript. LMW, JM, CTT, and DPW performed experiments and analyzed data. GP and MB performed metabolome experiments and analyzed data. PPN and THF gave conceptual and technological advice. JK conceived and designed the study and wrote the manuscript. JK is the guarantor of this work and, as such, had full access to all the data in the study and takes responsibility for the integrity of the data and the accuracy of the data analysis. 


\section{Acknowledgments}

The study was supported by grants from Deutsche Forschungsgemeinschaft (CRC 1118 and IRTG 1874/2 DIAMICOM). The authors thank Katrin Bennewitz for technical assistance in zebrafish histology and Karen Bieback for assistance in RT-qPCR. We thank the Metabolomics Core Technology Platform of the Excellence cluster "CellNetworks" (University of Heidelberg), and the Deutsche Forschungsgemeinschaft (grant ZUK 40/2010-3009262) for support with UPLC-based metabolite quantification. The authors acknowledge the support of the Core Facility Live Cell Imaging Mannheim at the Centre for Biomedicine and Medical Technology Mannheim (DFG INST 91027/10-1FUGG).

Address correspondence to: Jens Kroll, European Center for Angioscience (ECAS), Department of Vascular Biology and Tumor Angiogenesis, Medical Faculty Mannheim, Heidelberg University, Ludolf Krehl Str. 13-17, 68167 Mannheim, Germany. Phone: 49.0.621.383.71455; Email: jens.kroll@medma.uni-heidelberg.de.

1. Cho NH, et al. IDF Diabetes Atlas: Global estimates of diabetes prevalence for 2017 and projections for 2045. Diabetes Res Clin Pract. 2018;138:271-281.

2. Zimmet P, Alberti KG, Shaw J. Global and societal implications of the diabetes epidemic. Nature. 2001;414(6865):782-787.

3. Salunkhe VA, Veluthakal R, Kahn SE, Thurmond DC. Novel approaches to restore $\beta$ cell function in prediabetes and type 2 diabetes. Diabetologia. 2018;61(9):1895-1901

4. Boussageon R, et al. Effect of intensive glucose lowering treatment on all cause mortality, cardiovascular death, and microvascular events in type 2 diabetes: meta-analysis of randomised controlled trials. BMJ. 2011;343:d4169.

5. Thornalley PJ. Protein and nucleotide damage by glyoxal and methylglyoxal in physiological systems - role in ageing and disease. Drug Metabol Drug Interact. 2008;23(1-2):125-150.

6. Rabbani N, Thornalley PJ. Glyoxalase in diabetes, obesity and related disorders. Semin Cell Dev Biol. 2011;22(3):309-317.

7. Thornalley PJ. The glyoxalase system: new developments towards functional characterization of a metabolic pathway fundamental to biological life. Biochem J. 1990;269(1):1-11.

8. Vander Jagt DL, Hunsaker LA. Methylglyoxal metabolism and diabetic complications: roles of aldose reductase, glyoxalase-I, betaine aldehyde dehydrogenase and 2-oxoaldehyde dehydrogenase. Chem Biol Interact. 2003;143-144:341-351.

9. Baba SP, et al. Reductive metabolism of AGE precursors: a metabolic route for preventing AGE accumulation in cardiovascular tissue. Diabetes. 2009;58(11):2486-2497.

10. Morgenstern J, et al. Loss of glyoxalase 1 induces compensatory mechanism to achieve dicarbonyl detoxification in mammalian Schwann cells. J Biol Chem. 2017;292(8):3224-3238.

11. Schumacher D, et al. Compensatory mechanisms for methylglyoxal detoxification in experimental \& clinical diabetes. Mol Metab. 2018;18:143-152.

12. McLellan AC, Thornalley PJ, Benn J, Sonksen PH. Glyoxalase system in clinical diabetes mellitus and correlation with diabetic complications. Clin Sci. 1994;87(1):21-29.

13. Wang H, Meng QH, Gordon JR, Khandwala H, Wu L. Proinflammatory and proapoptotic effects of methylglyoxal on neutrophils from patients with type 2 diabetes mellitus. Clin Biochem. 2007;40(16-17):1232-1239.

14. Beisswenger PJ, Howell SK, Touchette AD, Lal S, Szwergold BS. Metformin reduces systemic methylglyoxal levels in type 2 diabetes. Diabetes. 1999;48(1):198-202.

15. Berner AK, et al. Protection against methylglyoxal-derived AGEs by regulation of glyoxalase 1 prevents retinal neuroglial and vasodegenerative pathology. Diabetologia. 2012;55(3):845-854.

16. Curtis TM, et al. Müller glial dysfunction during diabetic retinopathy in rats is linked to accumulation of advanced glycation end-products and advanced lipoxidation end-products. Diabetologia. 2011;54(3):690-698.

17. Zheng F, He C, Cai W, Hattori M, Steffes M, Vlassara H. Prevention of diabetic nephropathy in mice by a diet low in glycoxidation products. Diabetes Metab Res Rev. 2002;18(3):224-237.

18. Coughlan MT, et al. RAGE-induced cytosolic ROS promote mitochondrial superoxide generation in diabetes. $J$ Am Soc Nephrol. 2009;20(4):742-752.

19. Bierhaus A, et al. Methylglyoxal modification of Nav1.8 facilitates nociceptive neuron firing and causes hyperalgesia in diabetic neuropathy. Nat Med. 2012;18(6):926-933.

20. Riboulet-Chavey A, Pierron A, Durand I, Murdaca J, Giudicelli J, Van Obberghen E. Methylglyoxal impairs the insulin signaling pathways independently of the formation of intracellular reactive oxygen species. Diabetes. 2006;55(5):1289-1299.

21. Nigro C, et al. Methylglyoxal impairs endothelial insulin sensitivity both in vitro and in vivo. Diabetologia. 2014;57(7):1485-1494.

22. Fiory F, Lombardi A, Miele C, Giudicelli J, Beguinot F, Van Obberghen E. Methylglyoxal impairs insulin signalling and insulin action on glucose-induced insulin secretion in the pancreatic beta cell line INS-1E. Diabetologia. 2011;54(11):2941-2952.

23. Bo J, et al. Methylglyoxal impairs insulin secretion of pancreatic $\beta$-cells through increased production of ROS and mitochondrial dysfunction mediated by upregulation of UCP2 and MAPKs. J Diabetes Res. 2016;2016:2029854.

24. Dhar A, Dhar I, Jiang B, Desai KM, Wu L. Chronic methylglyoxal infusion by minipump causes pancreatic $\beta$-cell dysfunction and induces type 2 diabetes in Sprague-Dawley rats. Diabetes. 2011;60(3):899-908.

25. Moraru A, et al. Elevated levels of the reactive metabolite methylglyoxal recapitulate progression of type 2 diabetes. Cell Metab. 2018;27(4):926-934.e8.

26. Jang S, Kwon DM, Kwon K, Park C. Generation and characterization of mouse knockout for glyoxalase 1. Biochem Biophys Res Commun. 2017;490(2):460-465.

27. Heckler K, Kroll J. Zebrafish as a model for the study of microvascular complications of diabetes and their mechanisms. Int $J$ 
Mol Sci. 2017;18(9):E2002.

28. Wiggenhauser LM, Kroll J. Vascular damage in obesity and diabetes: Highlighting links between endothelial dysfunction and metabolic disease in zebrafish and man [published online ahead of print October 30, 2018]. Curr Vasc Pharmacol. https://doi.org $/ 10.2174 / 1570161116666181031101413$.

29. Gleeson M, Connaughton V, Arneson LS. Induction of hyperglycaemia in zebrafish (Danio rerio) leads to morphological changes in the retina. Acta Diabetol. 2007;44(3):157-163.

30. Olsen AS, Sarras MP, Intine RV. Limb regeneration is impaired in an adult zebrafish model of diabetes mellitus. Wound Repair Regen. 2010;18(5):532-542.

31. Dorsemans AC, et al. Impaired constitutive and regenerative neurogenesis in adult hyperglycemic zebrafish. J Comp Neurol. 2017;525(3):442-458.

32. Jörgens $\mathrm{K}$, et al. High tissue glucose alters intersomitic blood vessels in zebrafish via methylglyoxal targeting the VEGF receptor signaling cascade. Diabetes. 2015;64(1):213-225.

33. Hwang WY, et al. Efficient genome editing in zebrafish using a CRISPR-Cas system. Nat Biotechnol. 2013;31(3):227-229.

34. Wang H, Liu J, Wu L. Methylglyoxal-induced mitochondrial dysfunction in vascular smooth muscle cells. Biochem Pharmacol. 2009;77(11):1709-1716.

35. Sena CM, et al. Methylglyoxal promotes oxidative stress and endothelial dysfunction. Pharmacol Res. 2012;65(5):497-506.

36. Senyilmaz D, et al. Regulation of mitochondrial morphology and function by stearoylation of TFR1. Nature. 2015;525(7567):124-128.

37. Senyilmaz-Tiebe D, et al. Dietary stearic acid regulates mitochondria in vivo in humans. Nat Commun. 2018;9(1):3129.

38. Taneda S, Honda K, Tomidokoro K, Uto K, Nitta K, Oda H. Eicosapentaenoic acid restores diabetic tubular injury through regulating oxidative stress and mitochondrial apoptosis. Am J Physiol Renal Physiol. 2010;299(6):F1451-F1461.

39. Tocher DR. Metabolism and functions of lipids and fatty acids in teleost fish. Reviews in Fisheries Science. 2003;11(2):107-184

40. Giacco F, et al. Knockdown of glyoxalase 1 mimics diabetic nephropathy in nondiabetic mice. Diabetes. 2014;63(1):291-299.

41. Eames SC, Philipson LH, Prince VE, Kinkel MD. Blood sugar measurement in zebrafish reveals dynamics of glucose homeostasis. Zebrafish. 2010;7(2):205-213.

42. Landgraf K, et al. Short-term overfeeding of zebrafish with normal or high-fat diet as a model for the development of metabolically healthy versus unhealthy obesity. BMC Physiol. 2017;17(1):4.

43. Oka T, et al. Diet-induced obesity in zebrafish shares common pathophysiological pathways with mammalian obesity. $B M C$ Physiol. 2010;10:21.

44. Kumar GR, Babu DE. Effect of light, temperature and salinity on the growth of Artemia. Int J Eng Sci Invention. 2015;4:7-14

45. Frank RN. Diabetic retinopathy. N Engl J Med. 2004;350(1):48-58.

46. Wiggenhauser LM, Kohl K, Dietrich N, Hammes HP, Kroll J. Studying diabetes through the eyes of a fish: microdissection, visualization, and analysis of the adult tg(fli:EGFP) zebrafish retinal vasculature. $J$ Vis Exp. 2017;(130):56674.

47. Shah OJ, Wang Z, Hunter T. Inappropriate activation of the TSC/Rheb/mTOR/S6K cassette induces IRS1/2 depletion, insulin resistance, and cell survival deficiencies. Curr Biol. 2004;14(18):1650-1656.

48. Zhang J, Gao Z, Yin J, Quon MJ, Ye J. S6K directly phosphorylates IRS-1 on Ser-270 to promote insulin resistance in response to TNF-(alpha) signaling through IKK2. J Biol Chem. 2008;283(51):35375-35382.

49. Um SH, et al. Absence of S6K1 protects against age- and diet-induced obesity while enhancing insulin sensitivity. Nature. 2004;431(7005):200-205.

50. Maddison LA, Joest KE, Kammeyer RM, Chen W. Skeletal muscle insulin resistance in zebrafish induces alterations in $\beta$-cell number and glucose tolerance in an age- and diet-dependent manner. Am J Physiol Endocrinol Metab. 2015;308(8):E662-E669.

51. Maessen DE, Stehouwer CD, Schalkwijk CG. The role of methylglyoxal and the glyoxalase system in diabetes and other age-related diseases. Clin Sci. 2015;128(12):839-861.

52. Garrido D, et al. Fatty acid synthase cooperates with glyoxalase 1 to protect against sugar toxicity. PLoS Genet. 2015;11(2):e1004995.

53. Lawson ND, Weinstein BM. In vivo imaging of embryonic vascular development using transgenic zebrafish. Dev Biol. 2002;248(2):307-318.

54. Perner B, Englert C, Bollig F. The Wilms tumor genes wtla and wt1b control different steps during formation of the zebrafish pronephros. Dev Biol. 2007;309(1):87-96.

55. Kimmel CB, Ballard WW, Kimmel SR, Ullmann B, Schilling TF. Stages of embryonic development of the zebrafish. Dev Dyn. 1995;203(3):253-310.

56. Jao LE, Wente SR, Chen W. Efficient multiplex biallelic zebrafish genome editing using a CRISPR nuclease system. Proc Natl Acad Sci U S A. 2013;110(34):13904-13909.

57. Li M, Zhao L, Page-McCaw PS, Chen W. Zebrafish genome engineering using the CRISPR-Cas9 system. Trends Genet. 2016;32(12):815-827.

58. Hill JT, Demarest BL, Bisgrove BW, Su YC, Smith M, Yost HJ. Poly peak parser: Method and software for identification of unknown indels using sanger sequencing of polymerase chain reaction products. Dev Dyn. 2014;243(12):1632-1636.

59. Rabbani N, Thornalley PJ. Measurement of methylglyoxal by stable isotopic dilution analysis LC-MS/MS with corroborative prediction in physiological samples. Nat Protoc. 2014;9(8):1969-1979.

60. Thornalley PJ, Rabbani N. Assay of methylglyoxal and glyoxal and control of peroxidase interference. Biochem Soc Trans. 2014;42(2):504-510.

61. Clelland JD, Thornalley PJ. Synthesis of 14C-labelled methylglyoxal and S-D-lactoylglutathione. J Labelled Comp Radiopharm. 1990;28(12):1455-1464.

62. McLellan AC, Phillips SA, Thornalley PJ. Fluorimetric assay of D-lactate. Anal Biochem. 1992;206(1):12-16.

63. Rabbani N, Shaheen F, Anwar A, Masania J, Thornalley PJ. Assay of methylglyoxal-derived protein and nucleotide AGEs. Biochem Soc Trans. 2014;42(2):511-517.

64. McLellan AC, Thornalley PJ. Glyoxalase activity in human red blood cells fractioned by age. Mech Ageing Dev. 1989;48(1):63-71.

65. Skapare E, et al. Association of reduced glyoxalase 1 activity and painful peripheral diabetic neuropathy in type 1 and 2 diabetes 
mellitus patients. J Diabetes Complicat. 2013;27(3):262-267.

66. Srivastava S, Watowich SJ, Petrash JM, Srivastava SK, Bhatnagar A. Structural and kinetic determinants of aldehyde reduction by aldose reductase. Biochemistry. 1999;38(1):42-54.

67. Weger BD, et al. Extensive regulation of diurnal transcription and metabolism by glucocorticoids. PLoS Genet. 2016;12(12):e1006512

68. Zang L, Shimada Y, Nishimura Y, Tanaka T, Nishimura N. Repeated blood collection for blood tests in adult zebrafish. $J$ Vis Exp. 2015;(102):e53272.

69. Stoll SJ, Bartsch S, Augustin HG, Kroll J. The transcription factor HOXC9 regulates endothelial cell quiescence and vascular morphogenesis in zebrafish via inhibition of interleukin 8. Circ Res. 2011;108(11):1367-1377. 